\title{
On the physical structure of IRC +10216
}

\section{Ground-based and Herschel ${ }^{\star}$ observations of $\mathrm{CO}$ and $\mathrm{C}_{2} \mathrm{H}$}

\author{
E. De Beck ${ }^{1}$, R. Lombaert ${ }^{1}$, M. Agúndez ${ }^{2,3}$, F. Daniel ${ }^{2}$, L. Decin ${ }^{1,4}$, J. Cernicharo ${ }^{2}$, H. S. P. Müller ${ }^{5}$, M. Min ${ }^{6}$, \\ P. Royer ${ }^{1}$, B. Vandenbussche ${ }^{1}$, A. de Koter ${ }^{4,6}$, L. B. F. M. Waters ${ }^{4,7}$, M. A. T. Groenewegen ${ }^{8}$, M. J. Barlow ${ }^{9}$, \\ M. Guélin ${ }^{10,11}$, C. Kahane ${ }^{12}$, J. C. Pearson ${ }^{13}$, P. Encrenaz ${ }^{11}$, R. Szczerba ${ }^{14}$, and M. R. Schmidt ${ }^{14}$ \\ ${ }^{1}$ Institute for Astronomy, Department of Physics and Astronomy, KU Leuven, Celestijnenlaan 200D, 3001 Heverlee, Belgium \\ e-mail: elvire.debeck@ster.kuleuven.be \\ 2 CAB. INTA-CSIC. Crta Torrejón km 4, 28850 Torrejón de Ardoz, Madrid, Spain \\ ${ }^{3}$ LUTH, Observatoire de Paris-Meudon, 5 place Jules Janssen, 92190 Meudon, France \\ 4 Astronomical Institute "Anton Pannekoek", University of Amsterdam, Science Park 904, 1098 XH Amsterdam, The Netherlands \\ 5 I. Physikalisches Institut, Universität zu Köln, Zülpicher Str. 77, 50937 Köln, Germany \\ 6 Astronomical Institute Utrecht, University of Utrecht, PO Box 8000, 3508 TA Utrecht, The Netherlands \\ 7 SRON Netherlands Institute for Space Research, Sorbonnelaan 2, 3584 CA Utrecht, The Netherlands \\ 8 Royal Observatory of Belgium, Ringlaan 3, 1180 Brussels, Belgium \\ 9 Department of Physics and Astronomy, University College London, Gower Street, London WC1E 6BT, UK \\ 10 Institut de Radioastronomie Millimétrique (IRAM), 300 rue de la Piscine, 38406 Saint-Martin-dHères, France \\ 11 LERMA, CNRS UMR8112, Observatoire de Paris and École Normale Supérieure, 24 rue Lhomond, 75231 Paris Cedex 05, France \\ 12 LAOG, Observatoire de Grenoble, UMR 5571-CNRS, Université Joseph Fourier, Grenoble, France \\ 13 Jet Propulsion Laboratory, Caltech, Pasadena, CA 91109, USA \\ 14 N. Copernicus Astronomical Center, Rabianska 8, 87-100 Torun, Poland
}

Received 5 July 2011 / Accepted 9 January 2012

\section{ABSTRACT}

\begin{abstract}
Context. The carbon-rich asymptotic giant branch star IRC +10216 undergoes strong mass loss, and quasi-periodic enhancements of the density of the circumstellar matter have previously been reported. The star's circumstellar environment is a well-studied and complex astrochemical laboratory, in which many molecular species have been proved to be present. $\mathrm{CO}$ is ubiquitous in the circumstellar envelope, while emission from the ethynyl $\left(\mathrm{C}_{2} \mathrm{H}\right)$ radical is detected in a spatially confined shell around IRC +10216 . We recently detected unexpectedly strong emission from the $N=4-3,6-5,7-6,8-7$, and $9-8$ transitions of $\mathrm{C}_{2} \mathrm{H}$ with the IRAM $30 \mathrm{~m}$ telescope and with Herschel/HIFI, which challenges the available chemical and physical models.

Aims. We aim to constrain the physical properties of the circumstellar envelope of IRC +10216 , including the effect of episodic mass loss on the observed emission lines. In particular, we aim to determine the excitation region and conditions of $\mathrm{C}_{2} \mathrm{H}$ to explain the recent detections and to reconcile them with interferometric maps of the $N=1-0$ transition of $\mathrm{C}_{2} \mathrm{H}$.

Methods. Using radiative-transfer modelling, we provide a physical description of the circumstellar envelope of IRC +10216 , constrained by the spectral-energy distribution and a sample of 20 high-resolution and 29 low-resolution CO lines - to date, the largest modelled range of $\mathrm{CO}$ lines towards an evolved star. We furthermore present the most detailed radiative-transfer analysis of $\mathrm{C}_{2} \mathrm{H}$ that has been done so far.

Results. Assuming a distance of $150 \mathrm{pc}$ to IRC +10216 , the spectral-energy distribution was modelled with a stellar luminosity of $11300 L_{\odot}$ and a dust-mass-loss rate of $4.0 \times 10^{-8} M_{\odot} \mathrm{yr}^{-1}$. Based on the analysis of the 20 high-frequency-resolution CO observations, an average gas-mass-loss rate for the last 1000 years of $1.5 \times 10^{-5} M_{\odot} \mathrm{yr}^{-1}$ was derived. This results in a gas-to-dust-mass ratio of 375, typical for this type of star. The kinetic temperature throughout the circumstellar envelope is characterised by three power laws: $T_{\text {kin }}(r) \propto r^{-0.58}$ for radii $r \leq 9$ stellar radii, $T_{\text {kin }}(r) \propto r^{-0.40}$ for radii $9 \leq r \leq 65$ stellar radii, and $T_{\text {kin }}(r) \propto r^{-1.20}$ for radii $r \geq 65$ stellar radii. This model successfully describes all 49 observed CO lines. We also show the effect of density enhancements in the wind of IRC +10216 on the $\mathrm{C}_{2} \mathrm{H}$-abundance profile, and the close agreement we find of the model predictions with interferometric maps of the $\mathrm{C}_{2} \mathrm{H} \mathrm{N}=1-0$ transition and with the rotational lines observed with the IRAM $30 \mathrm{~m}$ telescope and Herschel/HIFI. We report on the importance of radiative pumping to the vibrationally excited levels of $\mathrm{C}_{2} \mathrm{H}$ and the significant effect this pumping mechanism has on the excitation of all levels of the $\mathrm{C}_{2} \mathrm{H}$-molecule.
\end{abstract}

Key words. stars: AGB and post-AGB - radiative transfer - astrochemistry - stars: mass-loss - stars: carbon stars: individual: IRC+10216

\section{Introduction}

The carbon-rich Mira-type star IRC +10216 (CW Leo) is located at the tip of the asymptotic giant branch (AGB), where

\footnotetext{
* Herschel is an ESA space observatory with science instruments provided by European-led Principal Investigator consortia and with important participation from NASA.
}

it loses mass at a high rate $\left(\sim 1-4 \times 10^{-5} M_{\odot} \mathrm{yr}^{-1}\right.$; Crosas \& Menten 1997; Groenewegen et al. 1998; Cernicharo et al. 2000; De Beck et al. 2010). Located at a distance of 120-250 pc (Loup et al. 1993; Crosas \& Menten 1997; Groenewegen et al. 1998; Cernicharo et al. 2000), it is the most nearby C-type AGB star. Additionaly, since its very dense circumstellar envelope (CSE) harbours a rich molecular chemistry, it has been deemed a prime 
carbon-rich AGB astrochemical laboratory. More than 70 molecular species have already been detected (e.g. Cernicharo et al. 2000; He et al. 2008; Tenenbaum et al. 2010), many of which are carbon chains, e.g. cyanopolyynes $\mathrm{HC}_{n} \mathrm{~N}(n=1,3,5,7$, $9,11)$ and $\mathrm{C}_{n} \mathrm{~N}(n=1,3,5)$. Furthermore, several anions have been identified, e.g. $\mathrm{C}_{n} \mathrm{H}^{-}(n=4,6,8$; Cernicharo et al. 2007; Remijan et al. 2007; Kawaguchi et al. 2007), and $\mathrm{C}_{3} \mathrm{~N}^{-}$ (Thaddeus et al. 2008), $\mathrm{C}_{5} \mathrm{~N}^{-}$(Cernicharo et al. 2008), and $\mathrm{CN}^{-}$(Agúndez et al. 2010). Detections towards IRC +10216 of acetylenic chain radicals $\left(\mathrm{C}_{n} \mathrm{H}\right)$, for $n=2$ up to $n=8$, have been reported by e.g. Guélin et al. (1978, $\left.\mathrm{C}_{4} \mathrm{H}\right)$, Cernicharo et al. (1986a,b, 1987b, $\left.\mathrm{C}_{5} \mathrm{H}\right)$, Cernicharo et al. (1987a) and Guélin et al. (1987, $\left.\mathrm{C}_{6} \mathrm{H}\right)$, Guélin et al. $\left(1997, \mathrm{C}_{7} \mathrm{H}\right)$, and Cernicharo \& Guélin (1996, $\left.\mathrm{C}_{8} \mathrm{H}\right)$.

The smallest $\mathrm{C}_{n} \mathrm{H}$ radical, ethynyl $\left(\mathrm{CCH}\right.$, or $\left.\mathrm{C}_{2} \mathrm{H}\right)$, was first detected by Tucker et al. (1974) in its $N=1-0$ transition in the interstellar medium (ISM) and in the envelope around IRC +10216 . It was shown to be one of the most abundant ISM molecules. The formation of $\mathrm{C}_{2} \mathrm{H}$ in the envelope of IRC +10216 is attributed mainly to photodissociation of $\mathrm{C}_{2} \mathrm{H}_{2}$ (acetylene), one of the most prominent molecules in carbonrich AGB stars. Fonfría et al. (2008) modelled $\mathrm{C}_{2} \mathrm{H}_{2}$ emission in the mid-infrared, which samples the dust-formation region in the inner CSE. The lack of a permanent dipole moment in the linearly symmetric $\mathrm{C}_{2} \mathrm{H}_{2}$-molecule implies the absence of pure rotational transitions that typically trace the outer parts of the envelope. $\mathrm{C}_{2} \mathrm{H}$, on the other hand, has prominent rotational lines that probe the chemical and physical conditions linked to $\mathrm{C}_{2} \mathrm{H}_{2}$ in these cold outer layers of the CSE. It has been established that $\mathrm{C}_{2} \mathrm{H}$ emission arises from a shell of radicals situated at $\sim 15^{\prime \prime}$ from the central star (Guélin et al. 1993). Observations with the IRAM $30 \mathrm{~m}$ telescope and Herschel/HIFI show strong emission in several high- $N$ rotational transitions of $\mathrm{C}_{2} \mathrm{H}$, something that is unexpected and challenges our understanding of this molecule in IRC + 10216 .

We present and discuss the high-sensitivity, high-resolution data obtained with the instruments on board Herschel (Pilbratt et al. 2010): HIFI (Heterodyne Instrument for the Far Infrared; de Graauw et al. 2010), SPIRE (Spectral and Photometric Imaging Receiver; Griffin et al. 2010), and PACS (Photodetector Array Camera and Spectrometer; Poglitsch et al. 2010), and with the IRAM $30 \mathrm{~m}^{\text {Telescope }}{ }^{1}$ in Sect. 2. The physical model for IRC +10216 's circumstellar envelope is presented and discussed in Sect. 3. The $\mathrm{C}_{2} \mathrm{H}$ molecule and our treatment of it is described in Sect. 4. A summary of our findings is provided in Sect. 5.

\section{Observations}

\subsection{Herschel/HIFI}

The HIFI data of IRC +10216 presented in this paper are part of a spectral line survey carried out with HIFI's wideband spectrometer (WBS; de Graauw et al. 2010) in May 2010, on three consecutive operational days (ODs) of the Herschel mission. The scan covers the frequency ranges $480-1250 \mathrm{GHz}$ and $1410-1910 \mathrm{GHz}$, with a spectral resolution of $1.1 \mathrm{MHz}$. All spectra were measured in dual beam switch (DBS; de Graauw et al. 2010) mode with a $3^{\prime}$ chop throw. This technique allows one to correct for any off-source signals in the spectrum, and to obtain a stable baseline.

\footnotetext{
${ }_{1}$ Based on observations carried out with the IRAM $30 \mathrm{~m}$ Telescope. IRAM is supported by INSU/CNRS (France), MPG (Germany) and IGN (Spain).
}

Since HIFI is a double-sideband (DSB) heterodyne instrument, the measured spectra contain lines pertaining to both the upper and the lower sideband. The observation and datareduction strategies disentangle these sidebands with very high accuracy, producing a final single-sideband (SSB) spectrum without ripples, ghost features, or any other instrumental effects, covering the spectral ranges mentioned above.

The two orthogonal receivers of HIFI (horizontal $\mathrm{H}$, and vertical V) were used simultaneously to acquire data for the whole spectral scan, except for band $2 \mathrm{a}^{2}$. Since we do not aim to study polarisation of the emission, we averaged the spectra from both polarisations, reducing the noise in the final product. This approach is justified since no significant differences between the $\mathrm{H}$ and V spectra are seen for the lines under study.

A detailed description of the observations and of the data reduction of this large survey is given by Cernicharo et al. (2010b) and Cernicharo et al. (in prep.). All $\mathrm{C}_{2} \mathrm{H}$ data in this paper are presented in the antenna temperature $\left(T_{\mathrm{A}}^{*}\right) \mathrm{scale}^{3}$. Roelfsema et al. (2012) describe the calibration of the instrument and mention uncertainties in the intensity of the order of $10 \%$.

For the current study we concentrate on observations of $\mathrm{CO}$ and $\mathrm{C}_{2} \mathrm{H}$. The ten $\mathrm{CO}$ transitions covered in the survey range from $J=5-4$ up to $J=11-10$ and from $J=14-13$ up to $J=16-15$. The $\mathrm{C}_{2} \mathrm{H}$ rotational transitions $N=6-5,7-6$, and $8-7$ are covered by the surveys in bands $1 \mathrm{a}(480-560 \mathrm{GHz})$, $1 \mathrm{~b}(560-640 \mathrm{GHz})$, and $2 \mathrm{a}(640-720 \mathrm{GHz})$, respectively. The $N=9-8$ transition is detected in band $2 \mathrm{~b}(720-800 \mathrm{GHz})$ of the survey, but with very low $\mathrm{S} / \mathrm{N}$; higher- $N$ transitions were not detected. A summary of the presented HIFI data of $\mathrm{C}_{2} \mathrm{H}$ is given in Table 1, the spectra are shown in Fig. 1.

\subsection{Herschel/SPIRE}

In the framework of the Herschel guaranteed time key programme "Mass loss of Evolved StarS" (MESS; Groenewegen et al. 2011) the SPIRE Fourier-transform spectrometer (FTS; Griffin et al. 2010) was used to obtain IRC +10216's spectrum on 19 November 2009 (OD 189). The SPIRE FTS measures the Fourier-transform of the source spectrum across two wavelength bands simultaneously. The short wavelength band (SSW) covers the range 194-313 $\mu \mathrm{m}$, while the long wavelength band (SLW) covers the range $303-671 \mu \mathrm{m}$. The total spectrum covers the 446-1575 GHz frequency range, with a final spectral resolution of $2.1 \mathrm{GHz}$. The quality of the acquired data permits the detection of lines as weak as 1-2 Jy. For the technical background and a description of the data-reduction process we refer to the discussions by Cernicharo et al. (2010a) and Decin et al. (2010b). These authors report uncertainties on the SPIRE FTS absolute fluxes of the order of 15-20\% for SSW data, 20-30\% for SLW data below $500 \mu \mathrm{m}$, and up to $50 \%$ for SLW data beyond $500 \mu \mathrm{m}$. We refer to Table 2 for a summary of the presented data, and show an instructive comparison between the HIFI and SPIRE data of the $\mathrm{C}_{2} \mathrm{H}$ lines $N=6-5$ up to $N=9-8$ in Fig. 2. Clearly, the lower resolution of the SPIRE spectrum causes many line blends in the spectra of AGB stars.

\footnotetext{
2 Because of an incompleteness, only the horizontal receiver was used during observations in band 2a. Supplementary observations will be executed later in the mission.

${ }^{3}$ The intensity in main-beam temperature $T_{\mathrm{MB}}$ is obtained via $T_{\mathrm{MB}}=$ $T_{\mathrm{A}}^{*} / \eta_{\mathrm{MB}}$, where $\eta_{\mathrm{MB}}$ is the main-beam efficiency, listed in Table 1 .
} 

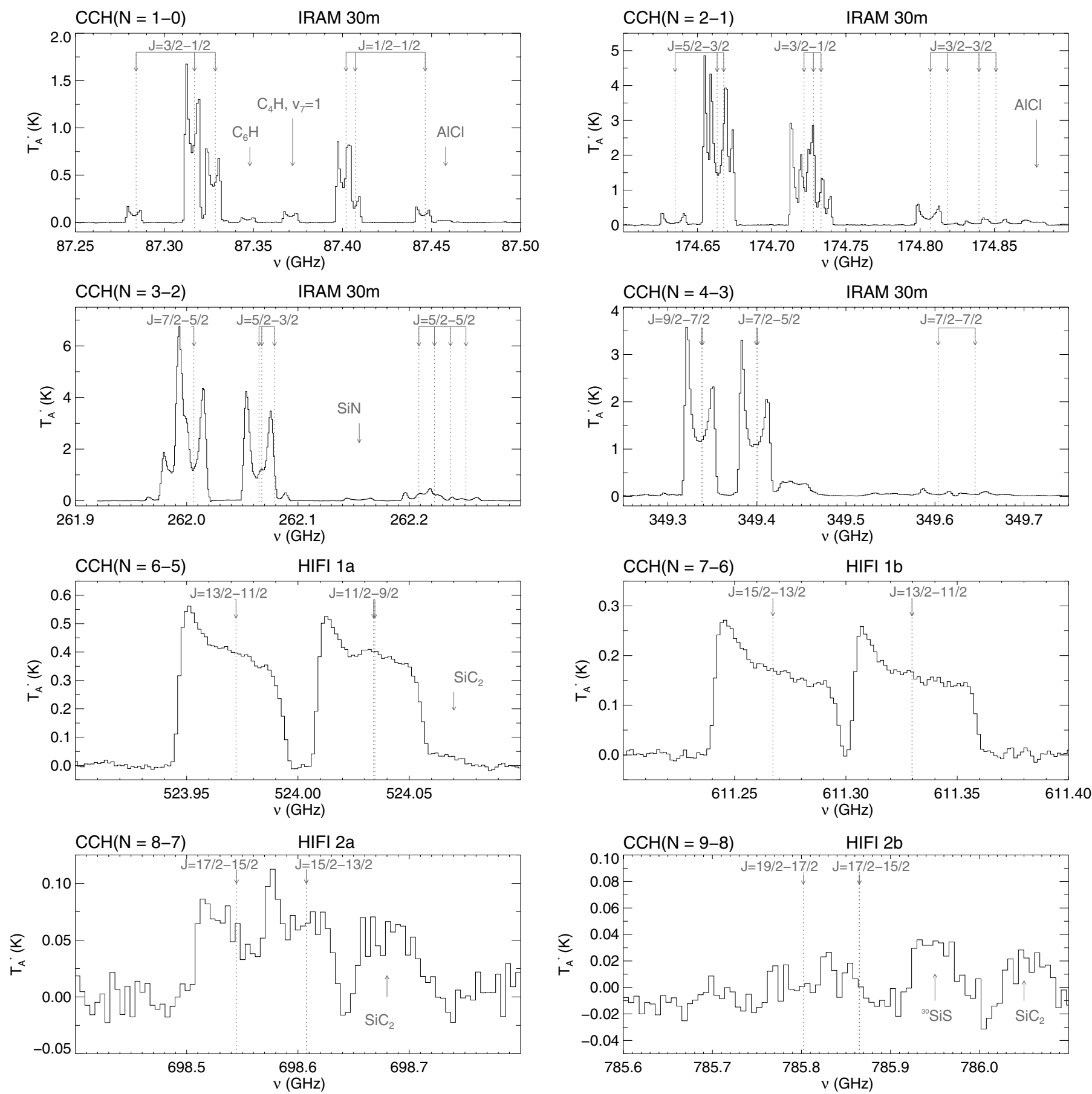

Fig. 1. $\mathrm{C}_{2} \mathrm{H} N=1-0,2-1,3-2,4-3,6-5,7-6,8-7$, and 9-8 rotational transitions in IRC +10216 's envelope as observed with the IRAM $30 \mathrm{~m}$ telescope and Herschel/HIFI. The fine structure components are labelled with the respective $J$-transitions in the top of each panel, while the hyperfine components are indicated with vertical dotted lines. For the sake of clarity, we omitted the components that are too weak to be detected. See Sect. 4 for details on the spectroscopic structure of $\mathrm{C}_{2} \mathrm{H}$. Additionally observed features related to other molecules are also identified.

\subsection{Herschel/PACS}

Decin et al. (2010b) presented PACS data of IRC +10216 , also obtained in the framework of the MESS programme (Groenewegen et al. 2011). The full data set consists of spectral energy distribution (SED) scans in the wavelength range $52-220 \mu \mathrm{m}$, obtained at different spatial pointings on 12 November 2009 (OD 182). We re-reduced this data set, taking into account not only the central spaxels of the detector, but all spaxels containing a contribution to the flux, i.e. 20 out of
25 spaxels in total. The here presented data set therefore reflects the total flux emitted by the observed regions with the assumption that there is no loss between the spaxels. The estimated uncertainty on the line fluxes is of the order of 30\%. Owing to instrumental effects, only the range $56-190 \mu \mathrm{m}$ of the PACS spectrum is usable. This range holds ${ }^{12} \mathrm{CO}$ transitions $J=14-13$ up to $J=42-41$, covering energy levels from $\sim 350 \mathrm{~cm}^{-1}$ up to $\sim 3450 \mathrm{~cm}^{-1}$. As is the case for the SPIRE data, line blends are present in the PACS spectrum due to the low spectral resolution of $0.08-0.7 \mathrm{GHz}$. 
Table 1. Summary of the IRAM $30 \mathrm{~m}$ and HIFI detections of $\mathrm{C}_{2} \mathrm{H}$.

\begin{tabular}{|c|c|c|c|c|c|c|c|c|c|}
\hline $\begin{array}{l}\text { Instrument } \\
\text { or band }\end{array}$ & $\eta_{\mathrm{MB}}$ & $\begin{array}{c}\text { HPBW } \\
\left({ }^{\prime \prime}\right)\end{array}$ & $\begin{array}{l}\text { Int. time } \\
(\min )\end{array}$ & $\begin{array}{l}\text { Noise } \\
(\mathrm{mK})\end{array}$ & $\begin{array}{c}\Delta v \\
(\mathrm{MHz})\end{array}$ & $\begin{array}{c}\Delta v \\
\left(\mathrm{~km} \mathrm{~s}^{-1}\right)\end{array}$ & $\begin{array}{c}\mathrm{CCH} \\
\text { transition }\end{array}$ & $\begin{array}{c}\text { Freq. range } \\
(\mathrm{MHz})\end{array}$ & $\begin{array}{c}I_{v, \mathrm{~A}}^{*} \\
(\mathrm{~K} \mathrm{MHz})\end{array}$ \\
\hline \multicolumn{10}{|l|}{ IRAM $30 \mathrm{~m}$} \\
\hline \multirow[t]{2}{*}{ A100/B100 } & 0.82 & 28.2 & 614 & 2.5 & 1.0 & 3.4 & $\begin{array}{l}N=1-0 \\
-3\end{array}$ & $872782-873396$ & 1500 \\
\hline & & & & & & & $J=1 / 2-1 / 2$ & $87392.8-87453.6$ & $\begin{array}{l}15.90 \\
7.58\end{array}$ \\
\hline \multirow[t]{4}{*}{ C150/D150 } & 0.68 & 14.1 & 215 & 10 & 1.0 & 1.7 & $N=2-1$ & & \\
\hline & & & & & & & $J=5 / 2-3 / 2$ & $174621.1-174682.5$ & 58.73 \\
\hline & & & & & & & $J=3 / 2-1 / 2$ & $174708.2-174744.9$ & 38.09 \\
\hline & & & & & & & $J=3 / 2-3 / 2$ & $174796.2-174863.7$ & 5.90 \\
\hline \multirow[t]{4}{*}{ E3 } & 0.57 & 9.4 & 161 & 10 & 1.0 & 1.1 & $N=3-2$ & & \\
\hline & & & & & & & $J=7 / 2-5 / 2$ & $261974.3-262023.4$ & 110.74 \\
\hline & & & & & & & $J=5 / 2-3 / 2$ & $262048.8-262084.4$ & 60.63 \\
\hline & & & & & & & $J=5 / 2-5 / 2$ & $262191.3-262270.0$ & 6.33 \\
\hline \multirow[t]{4}{*}{ E3 } & 0.35 & 7.0 & 79 & 5.3 & 2.0 & 1.7 & $N=4-3$ & & \\
\hline & & & & & & & $J=9 / 2-7 / 2$ & $349311.5-349368.3$ & 66.98 \\
\hline & & & & & & & $J=7 / 2-5 / 2$ & $349368.3-349421.6$ & 60.15 \\
\hline & & & & & & & $J=7 / 2-7 / 2$ & $349575.1-349671.8$ & 2.35 \\
\hline \multicolumn{10}{|l|}{ Herschel/HIFI } \\
\hline & 0.75 & 40.5 & 28 & 9.9 & 1.5 & 0.86 & $N=6-5$ & & \\
\hline & & & & & & & $J=13 / 2-11 / 2$ & $523941.9-524000.4$ & 18.60 \\
\hline & & & & & & & $J=11 / 2-9 / 2$ & $524000.4-524061.9$ & 18.74 \\
\hline \multirow{4}{*}{$1 b$} & 0.75 & 34.7 & 13 & 7.4 & 0.50 & 0.25 & $\begin{array}{c}J=11 / 2-11 / 2 \\
N=7-6\end{array}$ & - & - \\
\hline & & & & & & & $J=15 / 2-13 / 2$ & $611237.1-611301.6$ & 10.09 \\
\hline & & & & & & & $J=13 / 2-11 / 2$ & $611301.6-611364.1$ & 9.44 \\
\hline & & & & & & & $J=13 / 2-13 / 2$ & - & - \\
\hline \multirow[t]{4}{*}{$2 \mathrm{a}^{(\dagger)}$} & 0.75 & 30.4 & 12 & 13.7 & 4.5 & 1.8 & $N=8-7$ & & \\
\hline & & & & & & & $J=17 / 2-15 / 2$ & $698487.5-698619.0$ & 4.21 \\
\hline & & & & & & & $J=15 / 2-13 / 2$ & $698619.0-698738.0$ & 4.47 \\
\hline & & & & & & & $J=15 / 2-15 / 2$ & - & - \\
\hline \multirow[t]{4}{*}{$2 b$} & 0.75 & 27.0 & 15 & 10 & 6.0 & 2.2 & $N=9-8$ & & \\
\hline & & & & & & & $J=19 / 2-17 / 2$ & $785750.0-785875.0^{\ddagger}$ & $0.20^{\ddagger}$ \\
\hline & & & & & & & $J=17 / 2-15 / 2$ & $785875.0-785900.0^{\ddagger}$ & $0.20^{\ddagger}$ \\
\hline & & & & & & & $J=17 / 2-17 / 2$ & - & - \\
\hline
\end{tabular}

Notes. Columns are, respectively, the instrument or band with which we detected the $\mathrm{C}_{2} \mathrm{H}$ emission, the main-beam efficiency $\eta_{\mathrm{MB}}$, the half-power beam width HPBW, the integration time, the noise level in the spectra, the frequency resolution $\Delta v$, the velocity resolution $\Delta v$, the detected $\mathrm{C}_{2} \mathrm{H}$ rotational transitions, the frequency range in which we observed the lines, and the frequency-integrated intensity $I_{v, \mathrm{~A}}^{*}=\int T_{\mathrm{A}}^{*} \mathrm{~d} v$. ${ }^{(\dagger)}$ Only the horizontal polarisation was obtained. ${ }^{(*)}$ Owing to the low signal-to-noise ratio of this data set, these numbers are to be interpreted with caution.

Table 2. Summarised information on the SPIRE spectrum containing the $N=7-6$ transition of $\mathrm{C}_{2} \mathrm{H}$.

\begin{tabular}{lr|cr}
\hline \hline Frequency range & $446-1575 \mathrm{GHz}$ & Integration time & $2664 \mathrm{~s}$ \\
$\Delta v$ & $2.1 \mathrm{GHz}$ & $\Delta v$ & $201 \mathrm{~km} \mathrm{~s}^{-1}$ \\
$F_{v, \mathrm{~A}^{*}}$ & $16915 \mathrm{Jy} \mathrm{MHz}$ & $\sigma$ & $850 \mathrm{mJy}$ \\
\hline
\end{tabular}

Notes. $\Delta v$ is the frequency resolution, $\Delta v$ is the velocity resolution, and $\sigma$ is the rms noise. $F_{v, \mathrm{~A}^{*}}$ is the frequency-integrated flux in the given frequency range.

\subsection{IRAM $30 \mathrm{~m}$ : line surveys}

We combined the Herschel data with data obtained with the IRAM 30 m telescope at Pico Veleta. The $\mathrm{C}_{2} \mathrm{H} N=1-0$ transition was observed by Kahane et al. (1988); $N=2-1$ was observed by Cernicharo et al. (2000). $N=3-2$ and $N=4-3$ were observed between January and April 2010, using the EMIR receivers as described in detail by Kahane et al. (in prep.). The data-reduction process of these different data sets is described in detail in the listed papers. The observed $\mathrm{C}_{2} \mathrm{H}$ transitions are shown in Fig. 1, and details on the observations are listed in Table 1.

\section{The envelope model: dust and CO}

We assumed a distance $d=150 \mathrm{pc}$ to IRC +10216 , in good correspondence with literature values (Groenewegen et al. 1998; Men'shchikov et al. 2001; Schöier et al. 2007, and references therein). The second assumption in our models is that the effective temperature $T_{\text {eff }}=2330 \mathrm{~K}$, following the model of a large set of mid-IR lines of $\mathrm{C}_{2} \mathrm{H}_{2}$ and $\mathrm{HCN}$, presented by Fonfría et al. (2008). We determined the luminosity $L_{\star}$, the dust-massloss rate $\dot{M}_{\text {dust }}$, and the dust composition from a fit to the SED (Sect. 3.1). The kinetic temperature profile and the gas-massloss rate $\dot{M}$ were determined from a CO-line emission model (Sect. 3.2). The obtained envelope model will serve as the basis for the $\mathrm{C}_{2} \mathrm{H}$-modelling presented in Sect. 4. We point out that all modelling is performed in the radial dimension only, i.e. in 1D, assuming spherical symmetry throughout the CSE.

\subsection{Dust}

The dust modelling was performed using MCMax (Min et al. 2009), a Monte Carlo dust radiative transfer code. The best SED fit to the ISO SWS and LWS data, shown in Fig. 3, is based on a stellar luminosity $L_{\star}=11300 L_{\odot}$. This corresponds well with 
E. De Beck et al.: $\mathrm{CO}$ and $\mathrm{CCH}$ around $\mathrm{IRC}+10216$
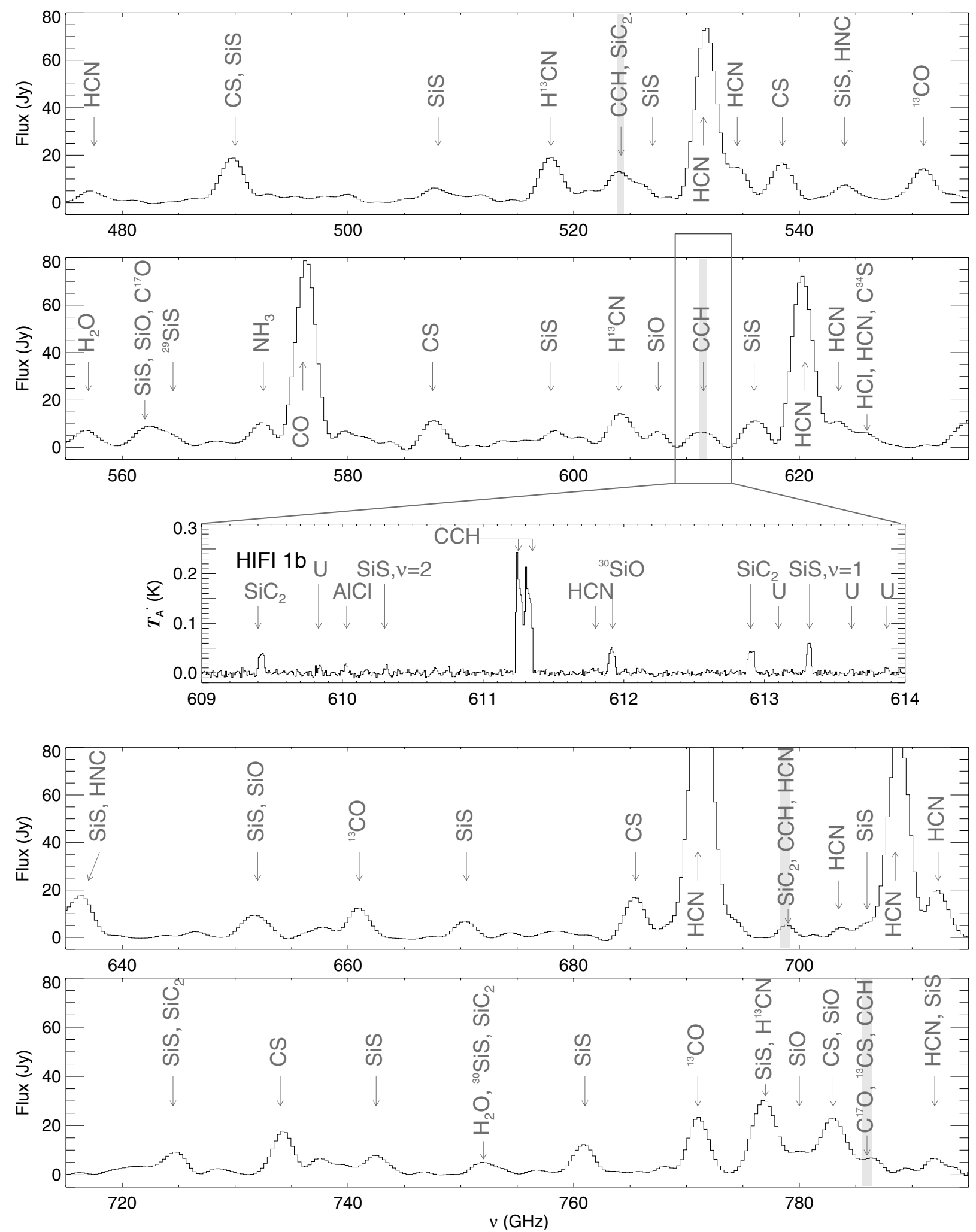

Fig. 2. SPIRE data in the range $475-795 \mathrm{GHz}$, containing the $\mathrm{C}_{2} \mathrm{H}$-lines detected with HIFI. The shaded strips in the panels indicate the spectral ranges of the HIFI data shown in the panels of Fig. 1. The labels mark the principal contributors to the main spectral features. A comparison of emission in the range 609-614 GHz, as obtained with SPIRE and with HIFI, is shown in the inset panel.

the results of Men'shchikov et al. $(2001)^{4}$, considering the difference in adopted distance. The combination of $L_{\star}$ and $T_{\text {eff }}$ gives a

\footnotetext{
4 The extensive modelling of Men'shchikov et al. was based on a lightcurve analysis and SED modelling, and holds a quoted uncertainty on the luminosity of $20 \%$.
}

stellar radius $R_{\star}$ of 20 milli-arcsec, which agrees very well with the values reported by e.g. Ridgway \& Keady (1988, 19 mas) and Monnier et al. (2000, 22 mas). 
Table 3. Dust composition in the CSE of IRC +10216 , used to produce the fit to the SED in Fig. 3.

\begin{tabular}{lccl}
\hline \hline Dust species & Shape & $\begin{array}{c}\text { Mass fraction } \\
(\%)\end{array}$ & References \\
\hline Amorphous carbon & DHS & 53 & Preibisch et al. (1993) \\
Silicon carbide & CDE & 25 & Pitman et al. (2008) \\
Magnesium sulfide & CDE & 22 & Begemann et al. (1994) \\
\hline
\end{tabular}

Notes. The columns list the dust species, the assumed shapes, the respective mass fractions, and the references to the optical constants of the different dust species.

IRC +10216 is a Mira-type pulsator, with a period of 649 days (Le Bertre 1992). Following Eq. (1) of Men'shchikov et al., we find that $L_{\star}$ varies between $L_{\star, \varphi=0} \approx 15800 L_{\odot}$ at maximum light (at phase $\varphi=0$ ), and $L_{\star, \varphi=0.5} \approx 6250 L_{\odot}$ at minimum light $(\varphi=0.5)$. Figure 3 shows the SED-variability corresponding to the $L_{\star}$-variability. Clearly, the spread on the photometric points can be accounted for by the models covering the full $L_{\star}$-range.

The adopted dust composition is given in Table 3. The main constituents are amorphous carbon $(\mathrm{aC})$, silicon carbide $(\mathrm{SiC})$, and magnesium sulfide (MgS), with mass fractions of $53 \%, 25 \%$, and $22 \%$, respectively. The aC grains are assumed to follow a distribution of hollow spheres (DHS; Min et al. 2003), with size $0.01 \mu \mathrm{m}$ and a filling factor of 0.8 . The population of the $\mathrm{SiC}$ and $\mathrm{MgS}$ dust grains is represented by a continuous distribution of ellipsoids (CDE; Bohren \& Huffman 1983), where the ellipsoids all have the volume of a sphere with radius $a_{\mathrm{d}}=0.1 \mu \mathrm{m}$. The CDE and DHS are believed to give a more realistic approximation of the characteristics of circumstellar dust grains than a population of spherical grains (Mie-particles). Assuming DHS for the dominant aC grains was found to provide the best general shape of the SED. All dust species are assumed to be in thermal contact.

The absorption and emission between $7 \mu \mathrm{m}$ and $10 \mu \mathrm{m}$ and around $14 \mu \mathrm{m}$ that is not fitted in our SED model can be explained by molecular bands of e.g. $\mathrm{HCN}$ and $\mathrm{C}_{2} \mathrm{H}_{2}$ (González-Alfonso \& Cernicharo 1999; Cernicharo et al. 1999).

Based on an average of the specific densities of the dust components $\rho_{\mathrm{s}}=2.41 \mathrm{~g} \mathrm{~cm}^{-3}$, we find a dust mass-loss rate of $4.0 \times 10^{-8} M_{\odot} \mathrm{yr}^{-1}$, with the dust density $\rho_{\text {dust }}(r)$, dust temperature $T_{\text {dust }}(r)$ and $Q_{\text {ext }} / a_{\mathrm{d}}$, with $Q_{\text {ext }}$ the total extinction efficiency, shown in Fig. 4. The inner radius of the dusty envelope, i.e. the dust condensation radius of the first dust species to be formed, is determined at $R_{\text {inner }}=2.7 R_{\star}$ by taking into account pressure-dependent condensation temperatures for the different dust species (Kama et al. 2009). These dust quantities are used as input for the gas radiative transfer, modelled in Sect. 3.2.

\section{2. $\mathrm{CO}$}

To constrain the gas kinetic temperature $T_{\text {kin }}(r)$ and the gas density $\rho_{\text {gas }}(r)$ throughout the envelope, we modelled the emission of 20 rotational transitions of ${ }^{12} \mathrm{CO}$ (Sect. 3.2), measured with ground-based telescopes and Herschel/HIFI. The gas radiative transfer was treated with the non-local thermal equilibrium (NLTE) code GASTRoNOoM (Decin et al. 2006, 2010c). To ensure consistency between the gas and dust radiative transfer models, we combined MCMax and GASTRoNOoM by passing on the dust properties (e.g. density and opacities) from the model presented in Sect. 3.1 and Fig. 4 to the gas modelling. The general

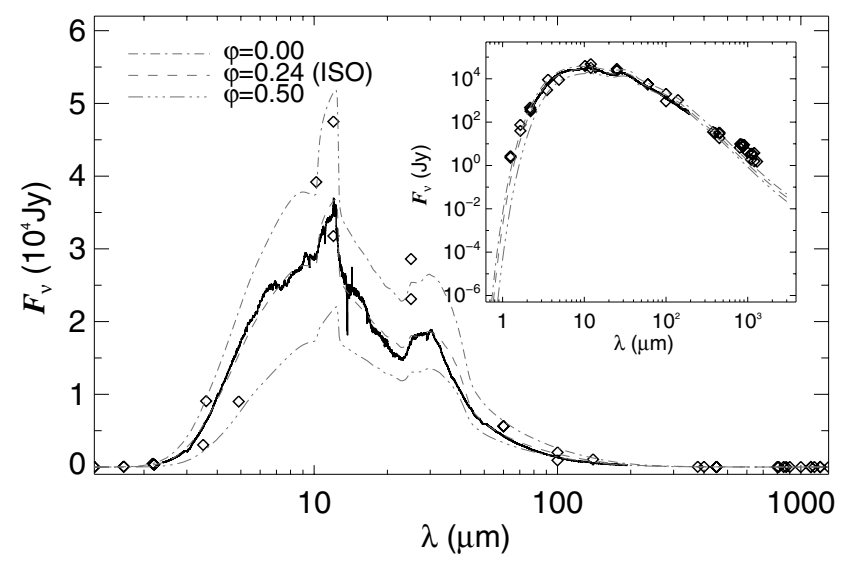

Fig. 3. SED models for IRC +10216 , using $\dot{M}_{\text {dust }}=4.0 \times 10^{-8} M_{\odot} \mathrm{yr}^{-1}$, and the dust composition and properties listed in Table 3 and spatial distribution and properties shown in Fig. 4. The SED model is constrained by the ISO SWS and LWS data (full black); photometric points (black diamonds) are taken from Ramstedt et al. (2008) and Ladjal et al. (2010). Dash-dotted grey: model at maximum light, with $15800 L_{\odot}$, dashed grey: best fit to the ISO data, at phase $\varphi=0.24$, using $11300 L_{\odot}$, dash-triple-dotted grey: model at minimum light, with $6250 L_{\odot}$. The inset is identical, but plotted in $\log -\log$ scale.
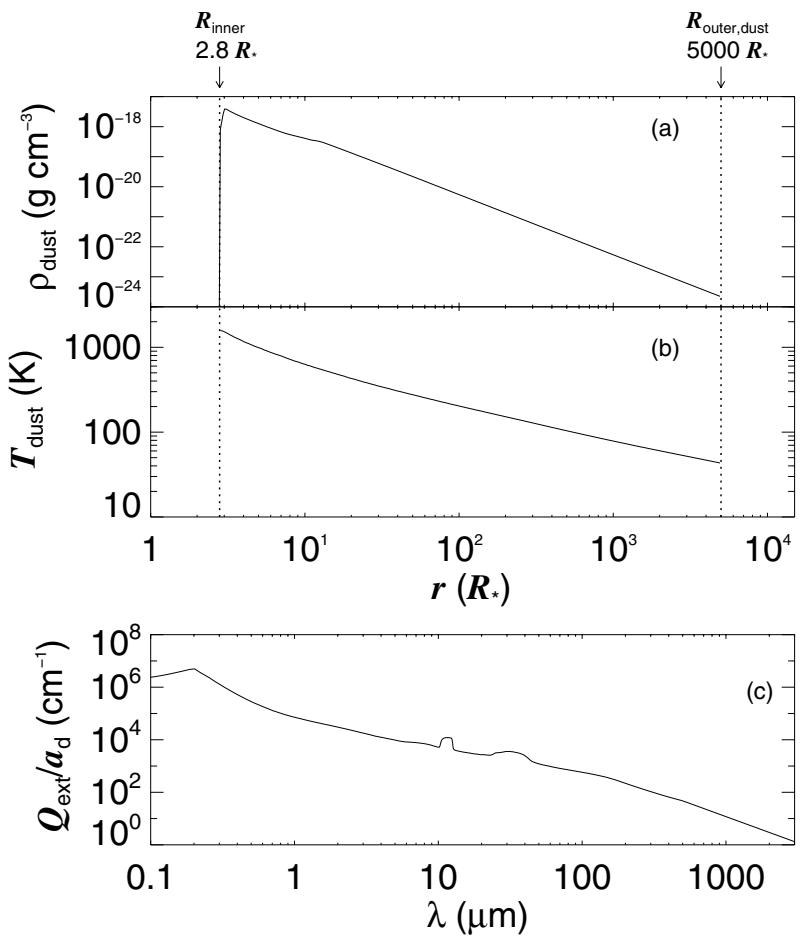

Fig. 4. Spatial distribution and properties of the dust for our best SED fit to the ISO data at $\varphi=0.24$ in Fig. 3 : a) dust density $\rho_{\text {dust }}(r)$, b) dust temperature $T_{\text {dust }}(r)$, and c) $Q_{\text {ext }} / a_{\mathrm{d}}$. The dashed lines indicate the inner and outer radii of the dusty envelope.

method behind this will be described in detail by Lombaert et al. (in prep.).

The large data set of high-spectral-resolution rotational transitions of $\mathrm{CO}$ consists of ten lines observed from the ground and ten lines observed with HIFI, which are listed in Table 4. Since the calibration of ground-based data is at times uncertain (Skinner et al. 1999), large data sets of lines that are observed simultaneously and with the same telescope and/or instrument are 


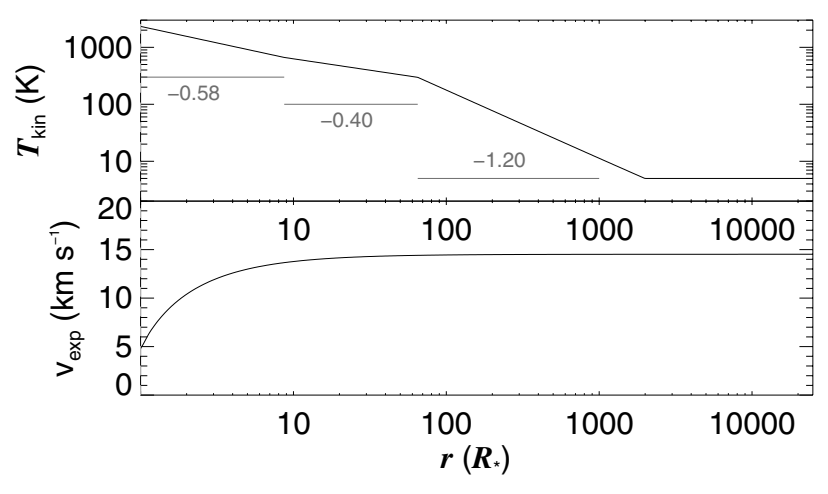

Fig. 5. Overview of the gas kinetic temperature and expansion velocity used in the radiative transfer model calculated with GASTRoNOoM. In the top panel, we indicate the exponents $\alpha$ from the $T_{\text {kin }}(r) \propto r^{\alpha}$-power laws used to describe the kinetic temperature, and the radial ranges they apply to. See Sect. 3.2.

of great value. Also, the observational uncertainties of the HIFI data are significantly lower than those of the data obtained with ground-based telescopes (20-40\%). Since the HIFI data of CO make up half of the available high-resolution lines in our sample, these will serve as the starting point for the gas modelling.

The adopted CO laboratory data are based on the work of Goorvitch \& Chackerian (1994) and Winnewisser et al. (1997), and are summarised in the Cologne Database for Molecular Spectroscopy (CDMS; Müller et al. 2005). Rotational levels $J=0$ up to $J=60$ are taken into account for both the ground vibrational state and the first excited vibrational state. $\mathrm{CO}-\mathrm{H}_{2}$ collisional rates were adopted from Larsson et al. (2002).

To reproduce the $\mathrm{CO}$ lines within the observational uncertainties, we used $L_{\star}=11300 L_{\odot}$, and a temperature profile ${ }^{5}$ that combines three power laws: $T_{\text {kin }}(r) \propto r^{-0.58}$ for $r \leq 9 R_{\star}$, $T_{\text {kin }}(r) \propto r^{-0.4}$ for $10 R_{\star} \leq r \leq 65 R_{\star}$, and $T_{\text {kin }}(r) \propto r^{-1.2}$ at larger radii, based on the work by Fonfría et al. (2008) and Decin et al. (2010a). The minimum temperature in the envelope is set to $5 \mathrm{~K}$. The radial profiles of $T_{\text {kin }}(r)$ and $v(r)$ are shown in Fig. 5. Using a fractional abundance $\mathrm{CO} / \mathrm{H}_{2}$ of $6 \times 10^{-4}$ in the inner wind, we find that a gas-mass-loss rate $\dot{M}$ of $1.5 \times 10^{-5} M_{\odot} \mathrm{yr}^{-1}$ reproduces the $\mathrm{CO}$ lines very well. Combining the results from Sect. 3.1 with those from the $\mathrm{CO}$ model, we find a gas-to-dustmass ratio of 375 , in the range of typical values for AGB stars $\left(10^{2}-10^{3}\right.$; e.g. Ramstedt et al. 2008).

The predicted $\mathrm{CO}$ line profiles are shown and compared to the observations in Fig. 6. All lines are reproduced very well in terms of integrated intensity, considering the respective observational uncertainties. The fraction of the predicted and observed velocity-integrated main-beam intensities $I_{\mathrm{MB} \text {,model }} / I_{\mathrm{MB} \text {,data }}$ varies in the range $74-145 \%$ for the groundbased data set, and in the range $89-109 \%$ for the HIFI data set. The shapes of all observed lines are also well reproduced, with the exception of the IRAM lines (possibly due to excitation and/or resolution effects).

Figure 7 shows the comparison between the observed CO transitions $J=14-13$ up to $J=42-41$ in the PACS spectrum and the modelled line profiles, convolved to the PACS resolution. Considering the uncertainty on the absolute data calibration and the presence of line blends with e.g. $\mathrm{HCN}, \mathrm{C}_{2} \mathrm{H}_{2}, \mathrm{SiO}, \mathrm{SiS}$, or $\mathrm{H}_{2} \mathrm{O}$ in several of the shown spectral ranges (Decin et al. 2010b), the overall fit of the CO lines is very good, with exception of the lines $J=14-13,15-14$, and 16-15. This significant difference

\footnotetext{
5 The central star is assumed to be a black body at a temperature $T_{\text {eff }}$.
}

Table 4. Overview of the transitions of $\mathrm{CO}$ and $\mathrm{C}_{2} \mathrm{H}$ shown throughout this paper.

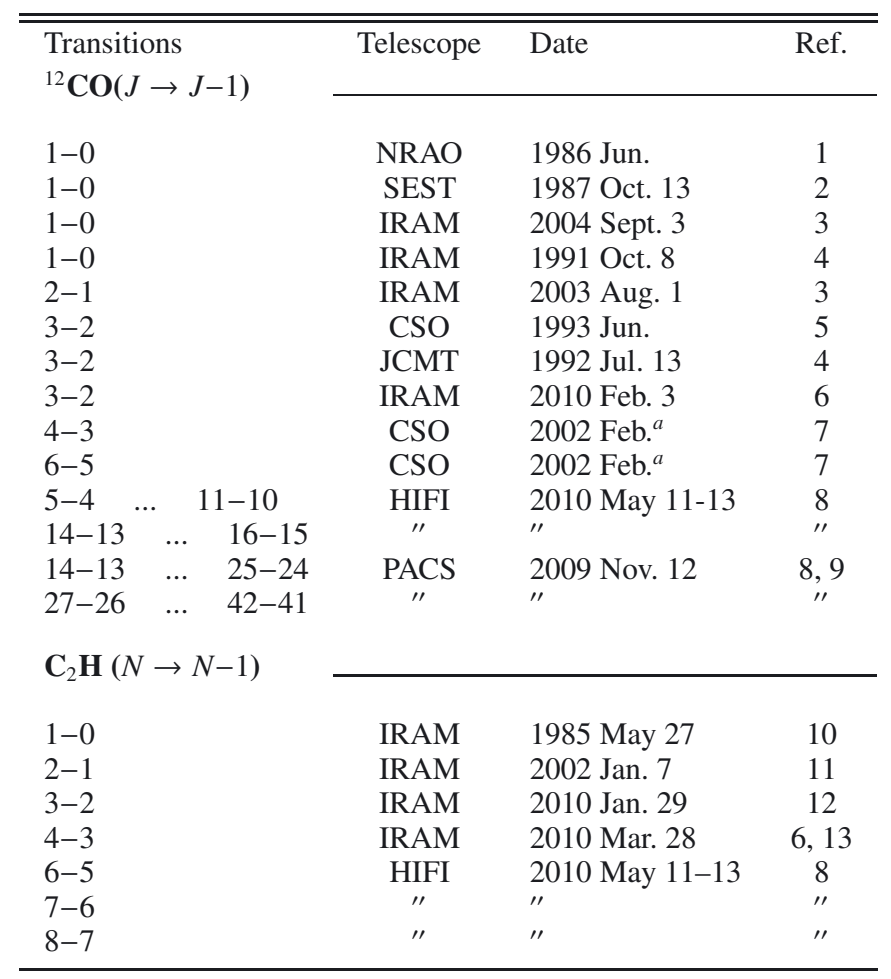

Notes. We list the transitions, the telescope/instrument, the dates of observation, and the literature references for previously published data. (a) Teyssier et al. (2006) state that observations were carried out between September 2001 and February 2002. A search of the CSO archive database resulted in observations in February 2002 that could be linked to their paper.

References. (1) Huggins et al. (1988); (2) Olofsson et al. (1993); (3) Cernicharo et al. (in prep.); (4) Groenewegen et al. (1996); (5) Wang et al. (1994); (6) Kahane et al. (in prep.); (7) Teyssier et al. (2006); (8) this paper; (9) Decin et al. (2010b); (10) Kahane et al. (1988); (11) Cernicharo et al. (2000); (12) Agúndez et al. (2010); (13) Cernicharo et al. (2011).

Table 5. Parameter set used to model the CO lines, as discussed in Sect. 3.2.

\begin{tabular}{ll|ll}
\hline \hline$d$ & $150 \mathrm{pc}$ & $\dot{M}_{\text {dust }}{ }^{a}$ & $4.0 \times 10^{-8} M_{\odot} \mathrm{yr}^{-1}$ \\
$L_{\star}{ }^{(a)}$ & $11300 L_{\odot}$ & $\dot{M}_{\text {gas }}$ & $1.5 \times 10^{-5} M_{\odot} \mathrm{yr}^{-1}$ \\
$R_{\star}$ & $4.55 \times 10^{13} \mathrm{~cm}$ & $\mathrm{CO} / \mathrm{H}_{2}$ & $6 \times 10^{-4}$ \\
& $\approx 655 R_{\odot}$ & $v_{\infty}$ & $14.5 \mathrm{~km} \mathrm{~s}^{-1}$ \\
$R_{\text {inner }}{ }^{(a)}$ & $2.7 R_{\star}$ & $v_{\text {turb }}$ & $1.5 \mathrm{~km} \mathrm{~s}^{-1}$ \\
$R_{\text {outer }}$ & $25000 R_{\star}$ & $T_{\text {kin }}(r)$ & $\propto r^{-0.58}\left(1 R_{\star} \leq r<9 R_{\star}\right)$ \\
$T_{\text {eff }}$ & $2330 \mathrm{~K}$ & & $\propto r^{-0.40}\left(9 R_{\star} \leq r<65 R_{\star}\right)$ \\
& & & $\propto r^{-1.20}\left(65 R_{\star} \leq r\right)$ \\
\hline
\end{tabular}

Notes. ${ }^{(a)}$ From SED modelling.

between the model predictions and the data could, to date, not be explained. Both the HIFI data and the higher- $J$ PACS lines are reproduced very well by the model.

Skinner et al. (1999) noted the possible presence of a gradient in the turbulent velocity $v_{\text {turb }}$, with decreasing values for increasing radial distance from the star. We find no clear evidence in our data set that this decrease in $v_{\text {turb }}$ is present. The model shown in Fig. 6 uses $v_{\text {turb }}=1.5 \mathrm{~km} \mathrm{~s}^{-1}$, and reproduces the line 

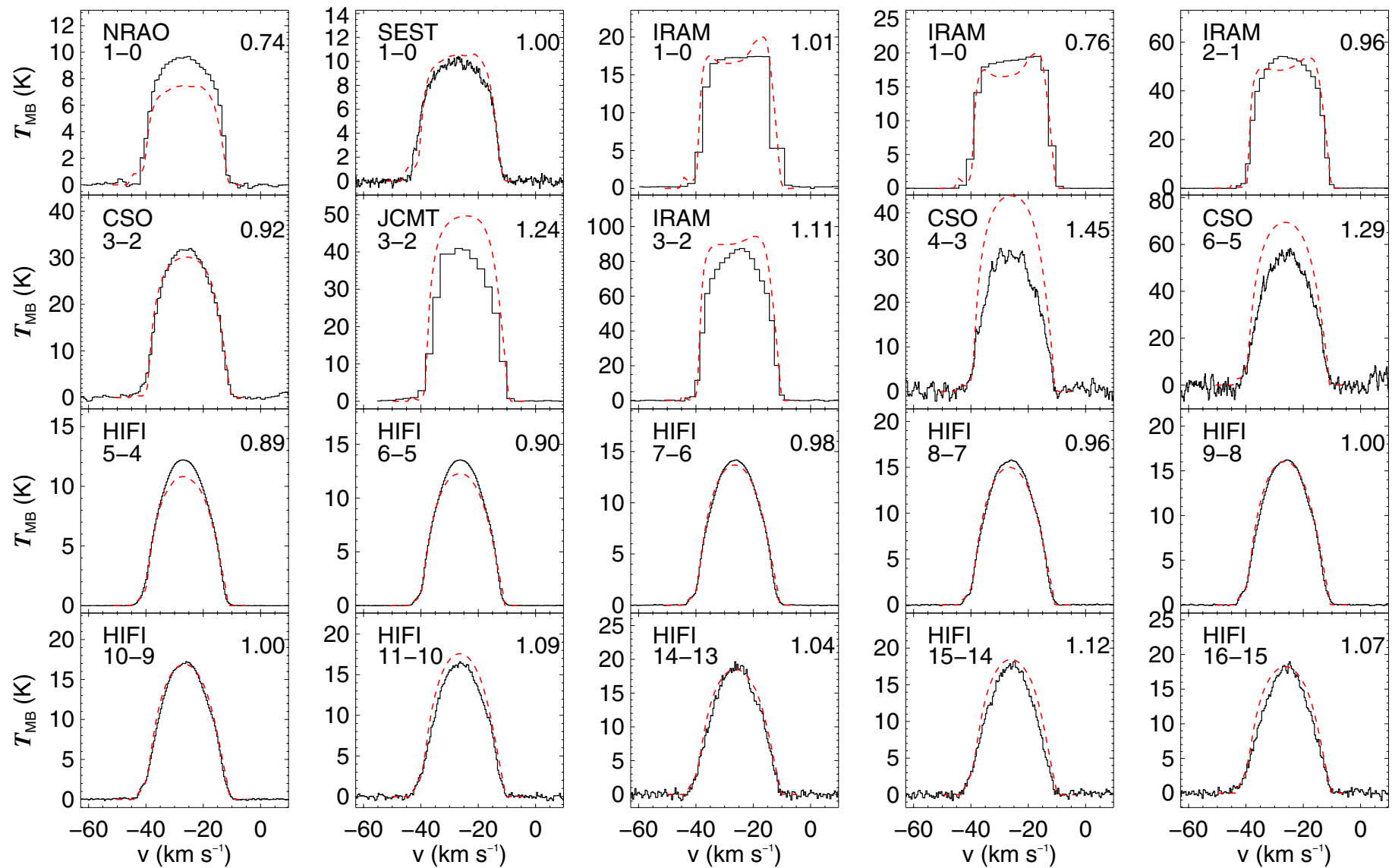

Fig. 6. Comparison of the observed ${ }^{12} \mathrm{CO}$ emission lines (black histogram), and the line profiles predicted by the GASTRoNOoM-model (red dashed line) with parameters as listed in Table 5. The line transitions $J-(J-1)$ and the telescopes with which they were observed are indicated in the upper left corner of every panel. The factor $I_{\mathrm{MB} \text {,model }} / I_{\mathrm{MB} \text {,data }}$ is given in the upper right corner of every panel.

shapes and intensities to a high degree. In Fig. 8 we compare the predictions of a set of CO-lines using different values for $v_{\text {turb }}$ in order to assess the influence of this parameter.

Assuming a lower value of $0.5 \mathrm{~km} \mathrm{~s}^{-1}$ or $1.0 \mathrm{~km} \mathrm{~s}^{-1}$ yields an incomplete reproduction of the emission profile between $-44 \mathrm{~km} \mathrm{~s}^{-1}$ and $-41 \mathrm{~km} \mathrm{~s}^{-1}$ for all lines observed with HIFI. Assuming a higher value of $2 \mathrm{~km} \mathrm{~s}^{-1}$ leads to the overprediction of the emission in this $v$-range for all observed lines, with the strongest effect for the lowest- $J$ lines. The influence of the different values of the turbulent velocity on the emission in the $v$-range of -41 to $-12 \mathrm{~km} \mathrm{~s}^{-1}$ is negligible.

\section{4. $\mathrm{C}_{2} \mathrm{H}$}

\subsection{Radical spectroscopy}

$\mathrm{C}_{2} \mathrm{H}(\bullet \mathrm{C} \equiv \mathrm{C}-\mathrm{H})$ is a linear molecule with an open shell configuration, i.e. it is a radical, with an electronic ${ }^{2} \Sigma^{+}$ground-state configuration (Müller et al. 2000). Spin-orbit coupling causes fine structure (FS), while electron-nucleus interaction results in hyperfine structure (HFS). Therefore, to fully describe $\mathrm{C}_{2} \mathrm{H}$ in its vibrational ground-state, we need the following coupling scheme:

$J=N+S$

$\boldsymbol{F}=\boldsymbol{J}+\boldsymbol{I}$,

where $N$ is the rotational angular momentum, not including the electron or quadrupolar angular momentum ( $\boldsymbol{S}$ and $\boldsymbol{I}$, respectively), $\boldsymbol{J}$ is the total rotational angular momentum, and $\boldsymbol{F}$ is the nuclear spin angular momentum.
The strong $\Delta J=\Delta N$ FS components have been detected up to $N=9-8$. Relative to the strong components, the weaker $\Delta J=$ 0 components decrease rapidly in intensity with increasing $N$, and they have been detected up to $N=4-3$. The HFS has also been (partially) resolved for transitions up to $N=4-3$.

Spectroscopic properties of the ground vibrational state of $\mathrm{C}_{2} \mathrm{H}$ have most recently been determined by Padovani et al. (2009) and are the basis for the entry in the CDMS.

Our treatment of the radiative transfer (Sect. 4.3) does not deal with the FS and HFS of $\mathrm{C}_{2} \mathrm{H}$, and is limited to the prediction of rotational lines $N \rightarrow N^{\prime}$ with $\Delta N=1$, according to a ${ }^{1} \Sigma$ approximation. Therefore, all levels treated are described with only one quantum number $N$. The final line profiles are calculated by splitting the total predicted intensity of the line over the different (hyper)fine components, depending on the relative strength of these components (CDMS), and preserving the total intensity. Note that, strictly speaking, this splitting is only valid under LTE conditions, but that, due to our approach of $\mathrm{C}_{2} \mathrm{H}$ as a ${ }^{1} \Sigma$-molecule, we will apply this scheme throughout this paper.

$\mathrm{C}_{2} \mathrm{H}$ has one bending mode $v_{2}$, and two stretching modes, $v_{1}$ and $v_{3}$. The rotational ground-state of the bending mode $\left(v_{2}\right)$ is situated $\sim 530 \mathrm{~K}$ above the ground state. The $\mathrm{C}-\mathrm{C}$ stretch $\left(v_{3}\right)$ at $\sim 2650 \mathrm{~K}$ is strong. The $\mathrm{C}-\mathrm{H}$ stretch $\left(v_{1}\right)$ at $\sim 4700 \mathrm{~K}$ is weak, and is resonant with the first excited ${ }^{2} \Pi$ electronic state at $\sim 5750 \mathrm{~K}$ of $\mathrm{C}_{2} \mathrm{H}$. For each of these vibrational states - groundstate, $v_{2}, v_{3}$, and $v_{1}$ - we consider 20 rotational levels, i.e. ranging from $N=0$ up to $N=19$.

The equilibrium dipole moments of the ground and first excited electronic states have been calculated as $\mu=0.769$ and 

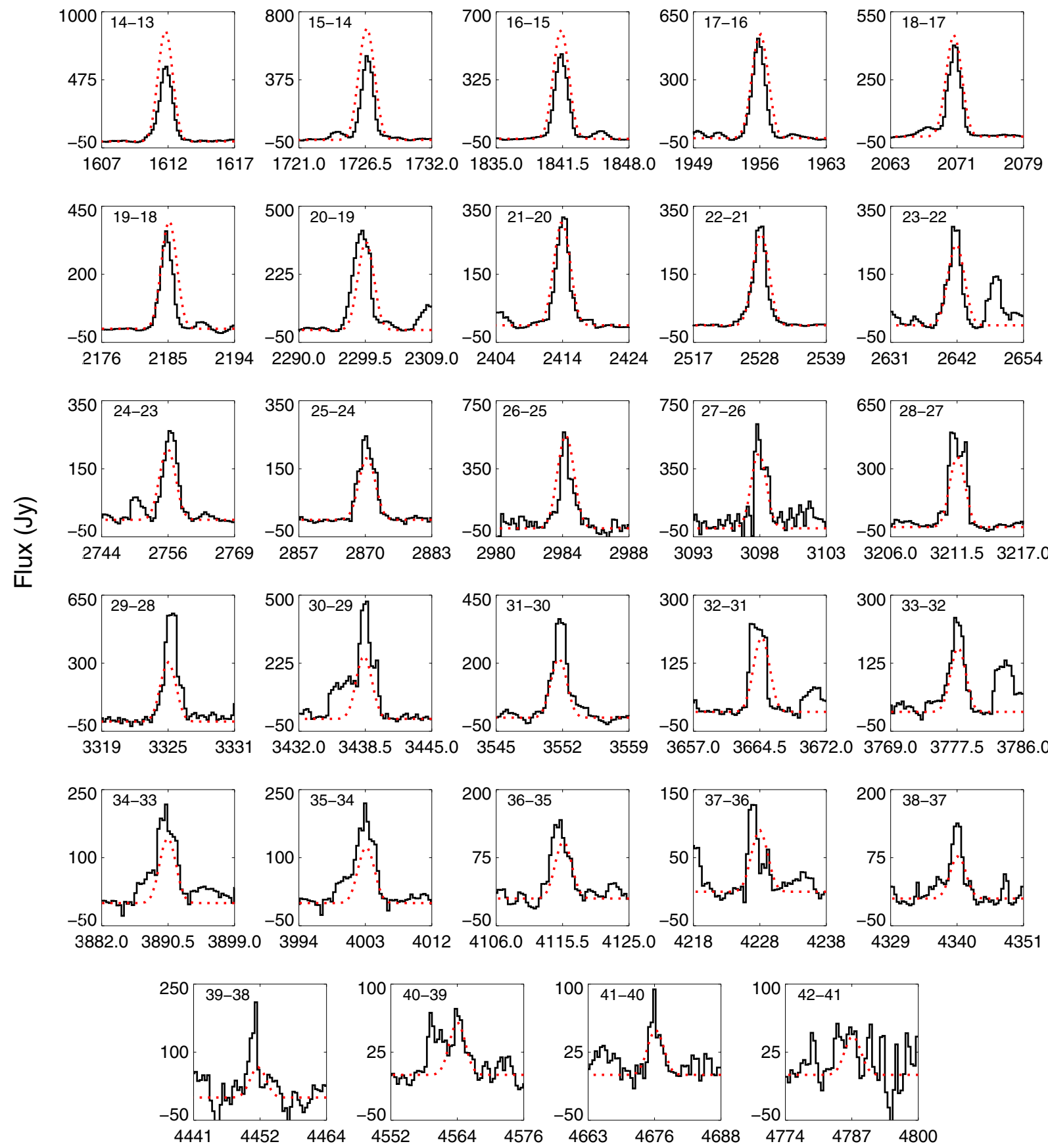

\section{$v(\mathrm{GHz})$}

Fig. 7. Comparison of CO-emission lines measured with PACS (black histogram) and the predicted line profiles (red dotted line). The transitions $J-(J-1)$ are labelled in the top left corner of each subpanel.

3.004 Debye, respectively (Woon 1995). The dipole moments of the fundamental vibrational states in the ground electronic state are also assumed to be 0.769 Debye. This is usually a good assumption as shown in the case of HCN by Deleon \& Muenter (1984). The band dipole moments for rovibrational transitions were calculated from infrared intensities published by Tarroni $\&$ Carter (2004): $\mu\left(v_{2}=1 \rightarrow 0\right)=0.110$ Debye, $\mu\left(v_{3}=1 \rightarrow\right.$ $0)=0.178$ Debye, and $\mu\left(v_{1}=1 \rightarrow 0\right)=0.050$ Debye. The influence of the vibrationally excited states on transitions in the vibrational ground state will be discussed in Sect. 4.3.
A more complete treatment of $\mathrm{C}_{2} \mathrm{H}$ will likely have to take into account, e.g., overtones of the $v_{2}$-state. These have nonnegligible intrinsic strengths (Tarroni \& Carter 2004) because of anharmonicity and vibronic coupling with the first excited electronic state. Spectroscopic data for several of these are already available in the CDMS. A multitude of high-lying states may also have to be considered because they have fairly high intrinsic intensities because of the vibronic interaction between the ground and the first excited electronic states. 

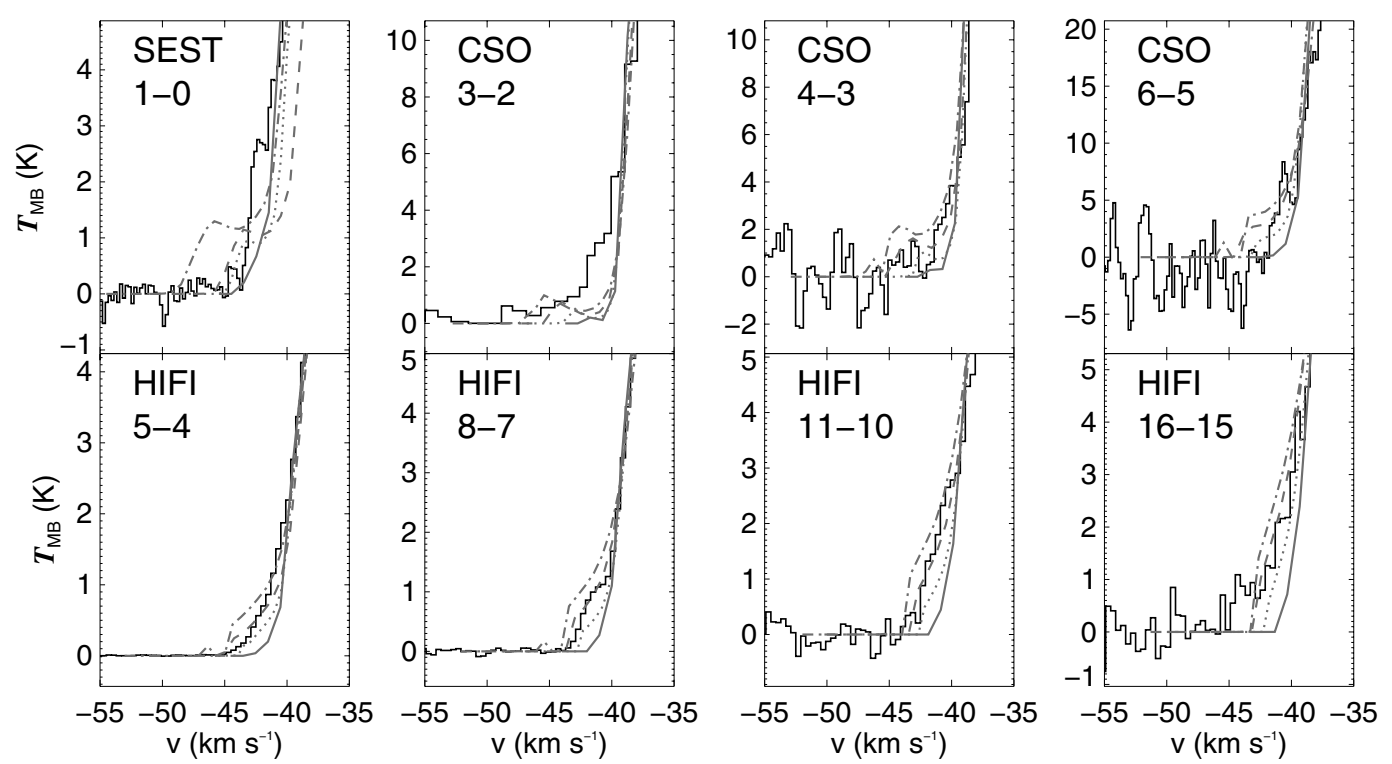

Fig. 8. Zoom on the blue wing of a selection of the CO line profiles, with the transitions $J-(J-1)$ labelled in the top left corner of each panel. We show the comparison between the observed lines and predicted lines with assumed values of $v_{\text {turb }}=0.5 \mathrm{~km} \mathrm{~s}^{-1}$ (full grey), $1.0 \mathrm{~km} \mathrm{~s}^{-1}$ (dotted grey), the adopted value of $1.5 \mathrm{~km} \mathrm{~s}^{-1}$ (dashed grey), and $2.0 \mathrm{~km} \mathrm{~s}^{-1}$ (dash-dotted grey).

Table 6. Properties of the transitions $N \rightarrow N^{\prime}$ used to model the $\mathrm{C}_{2} \mathrm{H}$ lines presented in Fig. 1.

\begin{tabular}{ccccccc}
\hline \hline$N$ & $N^{\prime}$ & $\begin{array}{c}v \\
(\mathrm{MHz})\end{array}$ & $\begin{array}{c}E_{N} / k \\
(\mathrm{~K})\end{array}$ & $\begin{array}{c}A_{N \rightarrow N^{\prime}} \\
\left(\mathrm{s}^{-1}\right)\end{array}$ & $S_{N \rightarrow N^{\prime}}$ & $g_{N}$ \\
\hline 1 & 0 & 87348.635 & 4.2 & $1.53 \times 10^{-6}$ & 1 & 3 \\
2 & 1 & 174694.734 & 12.6 & $1.47 \times 10^{-5}$ & 2 & 5 \\
3 & 2 & 262035.760 & 25.2 & $5.31 \times 10^{-5}$ & 3 & 7 \\
4 & 3 & 349369.178 & 41.9 & $1.30 \times 10^{-4}$ & 4 & 9 \\
6 & 5 & 524003.049 & 88.0 & $4.57 \times 10^{-4}$ & 6 & 13 \\
7 & 6 & 611298.435 & 117.4 & $7.34 \times 10^{-4}$ & 7 & 15 \\
8 & 7 & 698576.079 & 150.9 & $1.10 \times 10^{-3}$ & 8 & 17 \\
\hline
\end{tabular}

Notes. $v$ is the rest frequency of the transition, $E_{N} / k$ is the energy level of the upper level $N$ of the transition divided by the Boltzmann constant $k, A_{N \rightarrow N^{\prime}}$ is the Einstein- $A$ coefficient of the transition, $S_{N \rightarrow N^{\prime}}$ is the theoretical line strength of the line. $g_{N}$ is the statistical weight of the upper level $N$.

\subsection{Observational diagnostics}

The $\mathrm{C}_{2} \mathrm{H}$-emission doublets due to the molecule's fine structure (Sect. 4.1) are clearly present in all high-resolution spectra shown in Fig 1. For $N=1-0,2-1$, and 3-2, the hyperfine structure is clearly detected in our observations. The double-peaked line profiles are typical for spatially resolved optically thin emission (Olofsson, in Habing \& Olofsson 2003), and indicate that the emitting material has reached the full expansion velocity ${ }^{6}$, i.e. $v_{\infty}=14.5 \mathrm{~km} \mathrm{~s}^{-1}$. This is in accordance with the observed position of the emitting shells ( 16"; Guélin et al. 1999) and the velocity profile derived for this envelope (Sect. 3.2).

To derive information on the regime in which the lines are excited, we constructed a rotational diagram (Schloerb et al. 1983), using

$\frac{N}{Z\left(T_{\mathrm{rot}}\right)} \times \exp \left(-\frac{E_{u}}{k T_{\text {rot }}}\right)=\frac{3 k \times 10^{36}}{8 \pi^{3}} \frac{I_{v, \text { corr }}}{v S \mu^{2}}$,

\footnotetext{
${ }^{6}$ We assume that the half width at zero level of the CO emission lines, i.e. $14.5 \mathrm{~km} \mathrm{~s}^{-1}$, is indicative for the highest velocities reached by the gas particles in the CSE.
}

where $N$ is the column density, $Z$ the temperature dependent partition function, $E_{u}$ the energy of the upper level of the transition in $\mathrm{cm}^{-1}, k$ the Boltzmann constant in units of $\mathrm{cm}^{-1} \mathrm{~K}^{-1}, T_{\text {rot }}$ the rotational temperature in $\mathrm{K}, \mu$ the dipole moment in Debye, and $v$ the frequency of the transitions in Hz. $I_{v, \text { corr }}$ is the velocityintegrated antenna temperature,

$I_{v, \text { corr }}=\frac{1}{f_{\mathrm{bff}} \eta_{\mathrm{MB}}} \int_{v_{\mathrm{LSR}}-v_{\infty}}^{v_{\mathrm{LSR}}+v_{\infty}} T_{\mathrm{A}}^{*} \mathrm{~d} v$

corrected for the beam filling factor (Kramer 1997)

$$
f_{\text {bff }}= \begin{cases}1-\exp \left(-\left[\sqrt{\ln 2} \times \theta_{\text {source }} / \theta_{\text {beam }}\right]^{2}\right), & \theta_{\text {source }}<\theta_{\text {beam }} \\ 1, & \theta_{\text {source }} \geq \theta_{\text {beam }}\end{cases}
$$

and for the beam efficiency $\eta_{\mathrm{MB}}$ (see Table 1). We have assumed a uniform emission source with a size of $32^{\prime \prime}$ in diameter for all $\mathrm{C}_{2} \mathrm{H}$ transitions, based on the interferometric observations of the $N=1-0$ emission by Guélin et al. (1999).

From Fig. 9 we see that the $\mathrm{C}_{2} \mathrm{H}$ emission is characterised by a unique rotational temperature $T_{\text {rot }}=23.3( \pm 1.3) \mathrm{K}$, and a source-averaged column density $N=3.84( \pm 0.09) \times 10^{15} \mathrm{~cm}^{-2}$. The unique rotational temperature points to excitation of the lines in one single regime, in accordance with the abundance peak in a confined area that can be characterised with one gas temperature (Guélin et al. 1999).

\subsection{Radiative transfer modelling}

\subsubsection{Collisional rates}

For rotational transitions within the vibrational ground state and within the $v_{2}=1$ bending state and the $v_{1}=1$ and $v_{3}=1$ stretching states we adopted the recently published collisional rates of $\mathrm{HCN}-\mathrm{H}_{2}$ by Dumouchel et al. (2010). The lack of collisional rates for $\mathrm{C}_{2} \mathrm{H}$, and the similar molecular mass and size of $\mathrm{HCN}$ and $\mathrm{C}_{2} \mathrm{H}$ inspire this assumption. The collisional rates are given for 25 different temperatures between 5 and $500 \mathrm{~K}$, which 


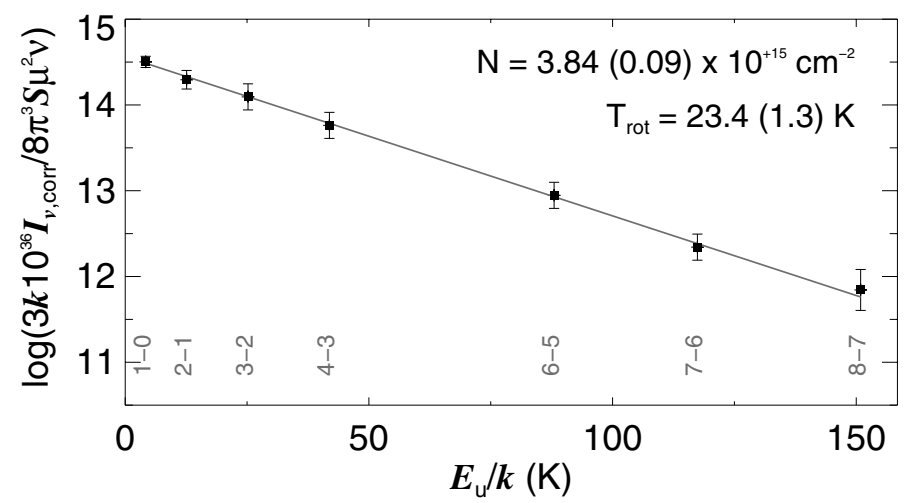

Fig. 9. Rotational diagram of the measured $\mathrm{C}_{2} \mathrm{H}$ transitions, based on Eq. (3). Transitions - in the ${ }^{1} \Sigma$ approximation - are labelled in grey. The numbers given in parentheses are the uncertainties on the derived column density and rotational temperature.

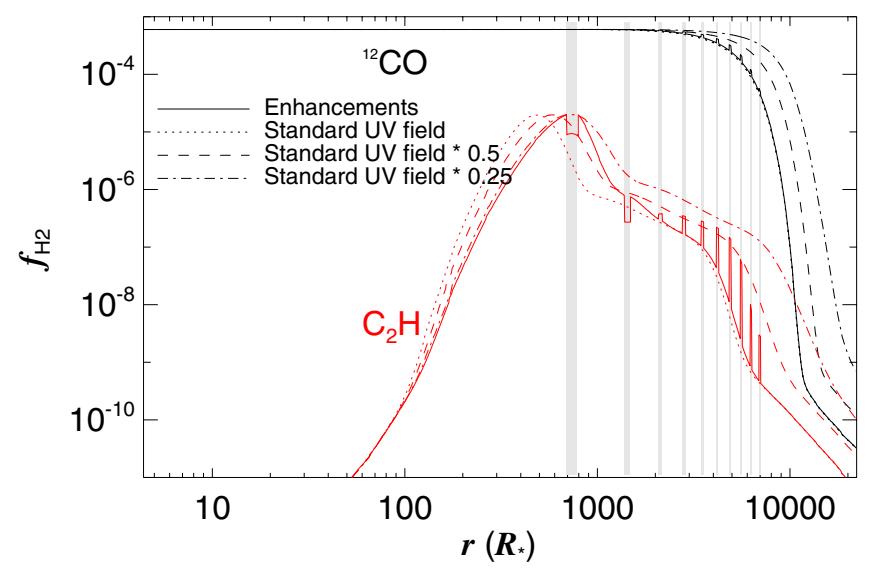

Fig. 10. Fractional abundance of ${ }^{12} \mathrm{CO}$ (black) and $\mathrm{C}_{2} \mathrm{H}$ (red) relative to $\mathrm{H}_{2}$ : model including the density enhancements (full lines), and models without density enhancements, assuming the average interstellar UV field of Draine (1978) scaled by a factor 1 (dotted lines), 0.5 (dashed lines), and 0.25 (dash-dotted lines). See Sect. 4.3.2 for a discussion of this plot.

covers the range of temperatures relevant for the $\mathrm{C}_{2} \mathrm{H}$ around IRC +10216 . Since $v_{3}$ and $v_{1}$ are at $\sim 2650 \mathrm{~K}$ and $\sim 4700 \mathrm{~K}$, respectively, collisional pumping to these vibrationally excited levels is unlikely for the low temperatures prevalent in the excitation region of $\mathrm{C}_{2} \mathrm{H}$, i.e. the radical shell at $\sim 16^{\prime \prime}$. Hence, for rovibrational transitions between the vibrationally excitated states $\left(v_{2}=1, v_{3}=1, v_{1}=1\right)$ and the vibrational ground state we adopted the same rates as for collisionally excited transitions within the vibrational ground state, but scaled down by a factor $10^{4}$, comparable to what has been done e.g. for $\mathrm{H}_{2} \mathrm{O}$ by Deguchi \& Nguyen-Q-Rieu (1990); Maercker et al. (2008) and Decin et al. (2010c). We did not consider collisionally excited transitions between the excited vibrational states.

We note that recent calculations by Dumouchel et al. (2010) have shown that the $\mathrm{HNC}$ collision rates at low temperatures differ by factors of a few from those of HCN. Since we may expect similar errors for $\mathrm{C}_{2} \mathrm{H}$, there is need for accurate $\mathrm{C}_{2} \mathrm{H}-\mathrm{H}_{2}$ collisional rates as well. The gas density, therefore, is not constrained better than this factor in the models presented in Sects. 3.2 and 4.3.2.

\subsubsection{Constraining the $\mathrm{C}_{2} \mathrm{H}$ abundance profile}

The calculation of the $\mathrm{C}_{2} \mathrm{H} / \mathrm{H}_{2}$ fractional abundance is based on the chemical model discussed by Agúndez et al. (2010), assuming the average interstellar UV field from Draine (1978), and a smooth envelope structure. The $\mathrm{CO}$ and $\mathrm{C}_{2} \mathrm{H}$ abundances corresponding to the envelope model presented in Sect. 3.2 are shown in Fig. 10.

The Plateau de Bure Interferometer (PdBI) maps of Guélin et al. (1999) show that the $3 \mathrm{~mm}$-lines of the three radicals $\mathrm{C}_{2} \mathrm{H}$, $\mathrm{C}_{4} \mathrm{H}$, and $\mathrm{C}_{6} \mathrm{H}$ have their brightness peaks at a radial angular distance of $\sim 16^{\prime \prime}$ from the central star. In Fig. 11a the contours of the $\mathrm{C}_{2} \mathrm{H}$ map by Guélin et al. (1999) are overlaid on the normalised brightness distribution of the $N=1-0$ transition predicted by a (one-dimensional) LTE model based on the abundance profile mentioned above. The extracted PdBI contours correspond to the velocity channel at the systemic velocity of IRC +10216 , representing the brightness distribution of the $\mathrm{C}_{2} \mathrm{H}$-transition in the plane of the sky. From Fig. 11a it is clear that this model leads to a brightness distribution that is concentrated too far inwards to agree with the shown PdBI contours.

Several authors have established that the mass-loss process of IRC +10216 shows a complex time-dependent behaviour. The images of Mauron \& Huggins (1999) and Leão et al. (2006) in dust-scattered stellar and ambient (optical) light, and the $\mathrm{CO}(J=1-0)$ maps of Fong et al. (2003) show enhancements in the dust and the gas density, with quasi-periodic behaviour. Recently, Decin et al. (2011) showed the presence of enhancements out to $\sim 320^{\prime \prime}$ based on PACS-photometry, consistent with the images of Mauron \& Huggins (1999).

Brown \& Millar (2003) presented a chemical envelope model incorporating the dust density enhancements observed by Mauron \& Huggins (1999). Cordiner \& Millar (2009) added density enhancements in the gas at the positions of the dust density enhancements. They assumed complete dust-gas coupling, based on the work by Dinh-V-Trung \& Lim (2008), who compared maps of the molecular shells of $\mathrm{HC}_{3} \mathrm{~N}$ and $\mathrm{HC}_{5} \mathrm{~N}$ with the images by Mauron \& Huggins (1999). To mimic these density enhancements in our model and evaluate the impact on the excitation and emission distribution of $\mathrm{C}_{2} \mathrm{H}$, we used the approximation of Cordiner \& Millar (2009), adding (to our "basic" model, presented in Sect. 3.2) ten shells of 2" width, with an intershell spacing of $12^{\prime \prime}$, starting at $14^{\prime \prime}$ from the central star. These shells - located at $14^{\prime \prime}-16^{\prime \prime}, 28^{\prime \prime}-30^{\prime \prime}$, etc. - are assumed to have been formed at gas-mass-loss rates of a factor six times the rate in the regions of "normal density", the intershell regions. We note that similar episodic mass loss was not added to the "basic" dust model presented in Sect. 3.1. The uncertainties on the data presented in Sect. 3.1 and the photometric variability of IRC +10216 are too large to constrain the expected small effect of the inclusion of these enhancements.

These density enhancements are included in the chemical model of Agúndez et al. (2010) by combining two regimes. Firstly, a model is run in which the chemical composition of a parcel of gas is followed as it expands in the envelope, considering an augmented extinction $\left(A_{V}\right)$ contribution from the density-enhanced shells located in the outer CSE. The density of the parcel is determined by the non-enhanced mass-loss rate $(\dot{M})$. A second model is run in which the composition of a density-enhanced shell is followed as it expands. The final abundance profile follows the abundance from the first model for the non-enhanced regions and follows the abundance of the second model for the density-enhanced shells. The resulting abundance profiles for ${ }^{12} \mathrm{CO}$ and $\mathrm{C}_{2} \mathrm{H}$ are compared to those corresponding 
to a smooth model without enhancements in Fig. 10. The ${ }^{12} \mathrm{CO}-$ abundance is affected by the inclusion of density enhancements only in the outer regions of the CSE. The impact on the radiativetransfer results for ${ }^{12} \mathrm{CO}$ is negligible, with differences in modelled integrated intensities of at most $3 \%$ compared to the model from Sect. 3.2.

We find that the presence of density enhancements significantly alters the abundance profile of $\mathrm{C}_{2} \mathrm{H}$, as was also discussed by Cordiner $\&$ Millar (2009). The abundance peak at $\sim 500 R_{\star}$ in the smooth model is shifted to $\sim 800 R_{\star}$ in the enhanced model, as shown in Fig. 10. This shift indicates that the photochemistry, in this particular case the photodissociation of $\mathrm{C}_{2} \mathrm{H}_{2}$, is initiated at larger radii than in the smooth model. This is caused by the stronger extinction due to the density enhancements in the outer envelope. At the position of the two innermost density enhancements the fractional $\mathrm{C}_{2} \mathrm{H}$ abundance is lower than in the intershell regions. The absolute abundance of $\mathrm{C}_{2} \mathrm{H}$ in these regions, however, is higher by a factor $2-3$ than in the neighbouring intershell regions. This is a combined effect of (1) the augmented shielding of $\mathrm{C}_{2} \mathrm{H}_{2}$ from incident interstellar UV radiation (which is no longer true for the outer shells), and (2) chemical effects such as faster reactions with e.g. $\mathrm{C}_{2} \mathrm{H}_{2}$ to form larger polyynes.

We note that the correspondence between the interferometric observations and the modelled number densities has significantly improved by including density enhancements (Fig. 11b). However, the peak intensity is located at somewhat too small radii compared to the observations. The adopted enhancements in the envelope are linked to $\dot{M}$-values of a factor six times as high as for the intershell regions, corresponding to the values used in the model of Cordiner \& Millar (2009). Increasing this factor or the number of shells in our model would increase the total mass of material shielding the $\mathrm{C}_{2} \mathrm{H}_{2}$ molecules from photodissociation by photons from the ambient UV field, and would hence shift the peak intensity of the predicted emission outwards. Furthermore, the presence of numerous dust arcs out to $\sim 320^{\prime \prime}$ is reported by Decin et al. (2011) and their effect will be included in future chemical models.

The "basic" model from Sects. 3.2 and 4.3 and the above introduced model with the density enhancements assume the interstellar UV field of Draine (1978). To assess the effect of a weaker UV field on the photodissociation of $\mathrm{C}_{2} \mathrm{H}_{2}$, and hence on the spatial extent of $\mathrm{C}_{2} \mathrm{H}$, we tested models assuming interstellar UV fields weaker by a factor of 2 and 4 . The corresponding $\mathrm{C}_{2} \mathrm{H}$ abundance profiles are shown in Fig. 10, and the modelled $N=1-0$ brightness distribution for the latter case is shown in Fig. 11c. Although the correspondence in this figure is very good, the $\mathrm{C}_{4} \mathrm{H}$ and $\mathrm{C}_{6} \mathrm{H}$ abundances produced by this chemical model cannot account for the coinciding brightness peaks of $\mathrm{C}_{2} \mathrm{H}, \mathrm{C}_{4} \mathrm{H}$, and $\mathrm{C}_{6} \mathrm{H}$ as reported by Guélin et al. (1999). In contrast, the density-enhanced model does reproduce this cospatial effect.

\subsubsection{Excitation analysis}

Assuming the $\mathrm{C}_{2} \mathrm{H}$ abundance obtained from the envelope model with density enhancements, we tested the influence of including the vibrational modes by modelling four cases: (1) including only the ground-state (GS) level; (2) including GS and $v_{2}$ levels, (3) including GS, $v_{2}$, and $v_{3}$ levels, and (4) including GS, $v_{2}, v_{3}$, and $v_{1}$ levels. An overview of the ratio of the predicted and the observed integrated intensities, $I_{\text {Model }} / I_{\text {Data }}$, for these four cases is shown in Fig. 12. The comparison of the line predictions for case (1) and case (4) under NLTE conditions is shown in Fig. 13.

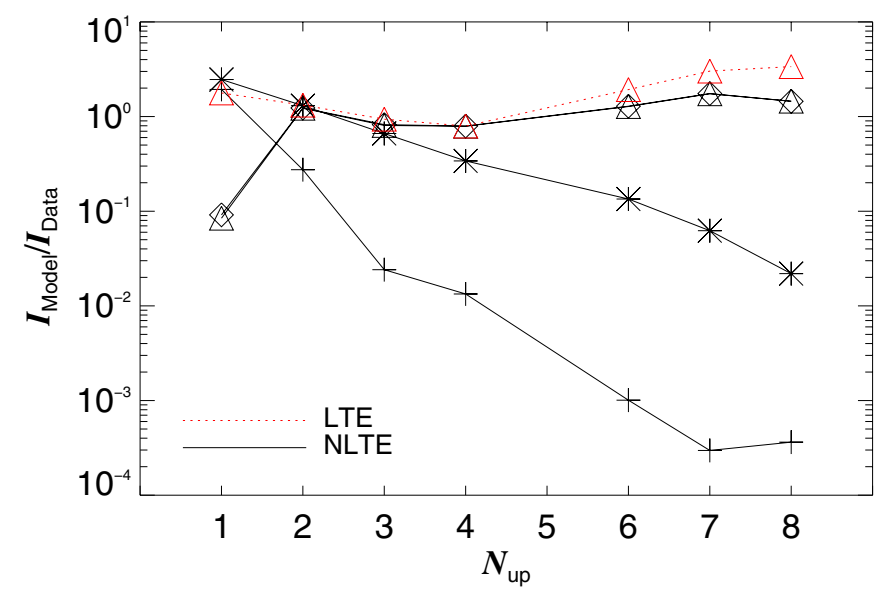

Fig. 12. Overview of the ratio $I_{\text {Model }} / I_{\text {Data }}$ of integrated intensities for the $\mathrm{C}_{2} \mathrm{H}$ lines in Fig. 1. The $x$-axis is labelled according to the upper $N$-level of the transition, $N_{\text {up }}$. Different ${ }^{1} \Sigma$-approximations of the $\mathrm{C}_{2} \mathrm{H}$ molecule are plotted with different symbols: crosses $(+)$ for the model including only the ground state (GS), asterisks (*) for the model including GS and the $v_{2}$ state, diamonds $(\diamond)$ for the model including GS, $v_{2}$, and $v_{3}$, and triangles $(\Delta)$ for the model including the GS and all three vibrationally excited states $v_{2}, v_{3}$, and $v_{1}$. Black symbols connected with full lines, and red symbols connected with dotted lines represent NLTE and LTE models, respectively.

Table 7. Einstein- $A$ coefficients (in $\mathrm{s}^{-1}$ ) for the transitions involving the $N=1$ and $N=3$ levels of the ground state (GS).

\begin{tabular}{llll}
\hline \hline & & \multicolumn{2}{c}{ Lower level } \\
& & GS, $N=3$ & GS, $N=1$ \\
\hline \multirow{3}{*}{ Upper level } & $v_{2}, N=2$ & $1.09 \times 10^{-1}$ & $8.16 \times 10^{-2}$ \\
& $v_{3}, N=2$ & $3.67 \times 10^{1}$ & $2.50 \times 10^{1}$ \\
& $v_{1}, N=2$ & $1.68 \times 10^{1}$ & $1.13 \times 10^{1}$ \\
\hline
\end{tabular}

Under LTE conditions, $I_{\text {Model }} / I_{\text {Data }}$ for a given transition is the same for all four cases, indicating that the vibrationally excited states are not populated under the prevailing gas kinetic temperatures of $\sim 20 \mathrm{~K}$ in the radical shell. Under NLTE conditions, however, the predicted line intensities are very sensitive to the inclusion of the vibrationally excited states. In the two cases where the $v_{3}$-state is included, the predicted intensity of the $N=1-0$ transition is only $9 \%$ of the observed value, while transitions with $N_{\text {up }} \geq 4$ are more easily excited. This is linked to the involved Einstein- $A$ coefficients. For example, Table 7 gives the Einstein- $A$ coefficients of the transitions involving the $N=2$ level of the vibrationally excited states and the $N=3$ and $N=1$ levels of the ground state. The higher values of the Einstein- $A$ coefficients for transitions to the $N=3$ level lead to a more effective population of this level than of the $N=1$ level. In particular, when including $v_{3}$ and $v_{1}$, this causes an underprediction of the intensity of the $N=1-0$ ground state transition, while this effect is insignificant when only $v_{2}$ is included. A visualisation of this scheme is shown in Fig. 14. This pumping mechanism is not limited to $N=3$, but also affects higher levels, explaining the easier excitation of the transitions with $N_{\text {up }} \geq 4$ mentioned before.

\subsubsection{Turbulent velocity}

As for $\mathrm{CO}$, we tested the influence of $v_{\text {turb }}$ on the predicted $\mathrm{C}_{2} \mathrm{H}$-emission, considering values $v_{\text {turb }}=0.5,1.0$, 1.5 , and $2.0 \mathrm{~km} \mathrm{~s}^{-1}$. We find that the predicted line intensity 


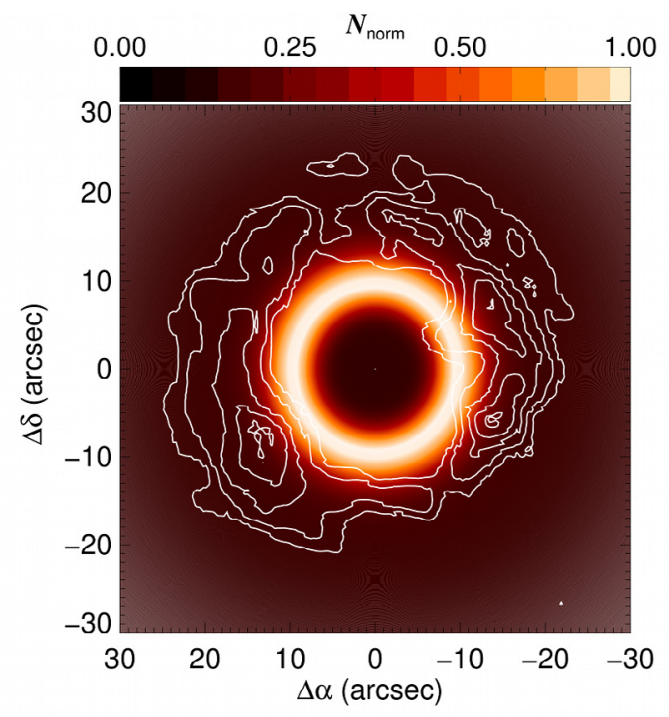

(a) Chemical model assuming time-independent mass loss

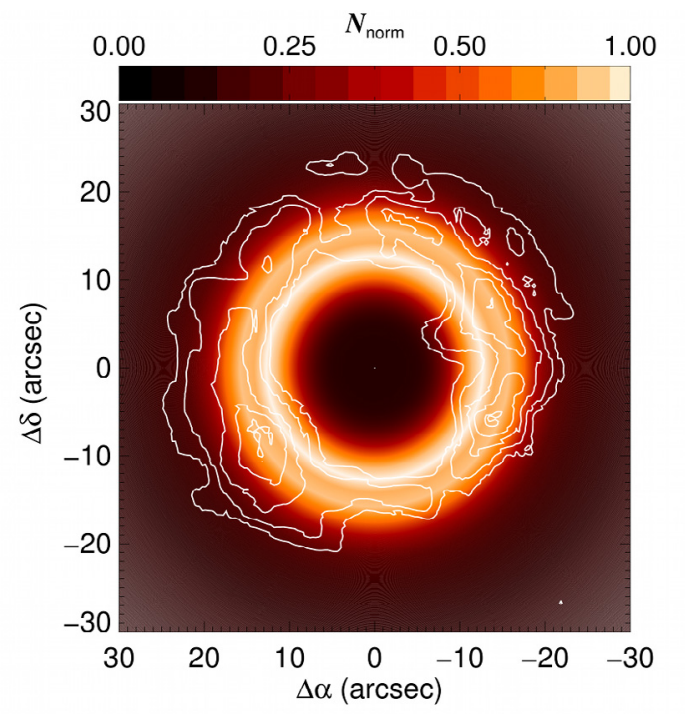

(b) Chemical model assuming time-dependent mass loss

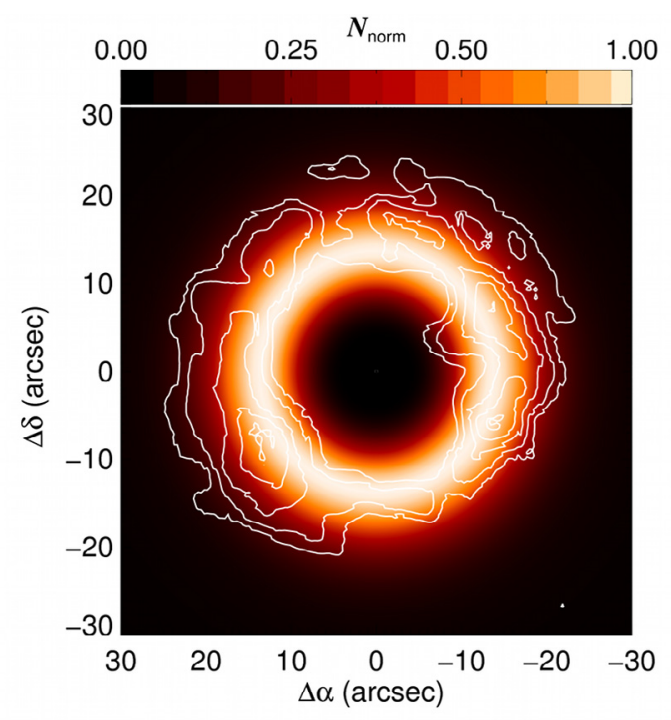

(c) Chemical model assuming time-independent mass loss and an interstellar UV field a quarter as strong as in the standard model presented in panel (a)

Fig. 11. Contours (in white) of the brightness distribution of the $\mathrm{C}_{2} \mathrm{H} N=1-0$ transition measured by Guélin et al. (1999) with PdBI, overlayed on the normalised predicted brightness distribution, assuming LTE conditions. The three panels represent the results obtained with three different chemical models. For the map in panel a), we assumed a constant mass loss, and the interstellar UV field of Draine (1978). The map in panel b) shows the brightness distribution when assuming density enhancements in the CSE as described by Cordiner \& Millar (2009) and as discussed in Sect. 4.3.2. For panel c), we assumed again a constant mass loss, but an interstellar UV field that is only $25 \%$ as strong as that presented by Draine (1978).

increases ${ }^{7}$ with $25-55 \%$ when $v_{\text {turb }}$ is increased from $0.5 \mathrm{~km} \mathrm{~s}^{-1}$ to $2.0 \mathrm{~km} \mathrm{~s}^{-1}$. From the comparison of the predicted and observed shapes of the emission lines, we conclude that values of $v_{\text {turb }}$ in the range $0.5-1.5 \mathrm{~km} \mathrm{~s}^{-1}$ give the best results. This is consistent with our discussion in Sect. 3.2 for the CO-lines, and with values generally used for AGB envelopes (Skinner et al. 1999).

\footnotetext{
7 This is valid for all lines, except for the $N=1-0$ transition, where the predicted intensity decreases by $40 \%$.
}

\subsection{Vibrationally excited states}

The removal of a hydrogen atom from $\mathrm{C}_{2} \mathrm{H}_{2}$ through photodissociation causes the produced $\mathrm{C}_{2} \mathrm{H}$ molecule to be bent, rather than linear (Mordaunt et al. 1998). This means that this formation route of $\mathrm{C}_{2} \mathrm{H}$ favours the population of the $v_{2}=1$ state over the population of the ground state. However, the Einstein- $A$ coefficients for rovibrational transitions in the band $v_{2}=1 \rightarrow 0$ are in the range $0.01-1 \mathrm{~s}^{-1}$, and by far exceed the photodissociation rates of $\mathrm{C}_{2} \mathrm{H}_{2}$, which are of the order of $10^{-9} \mathrm{~s}^{-1}$ 

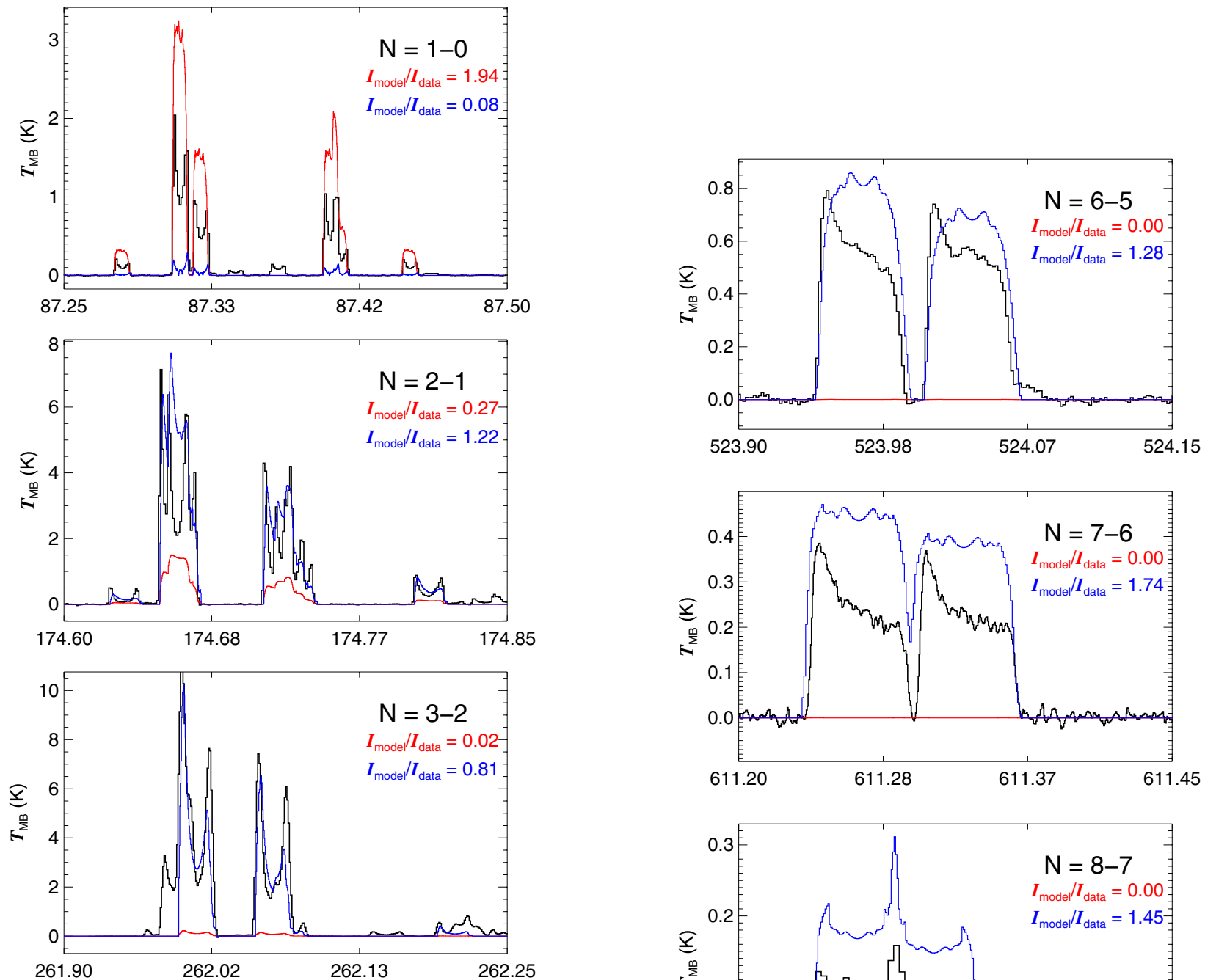

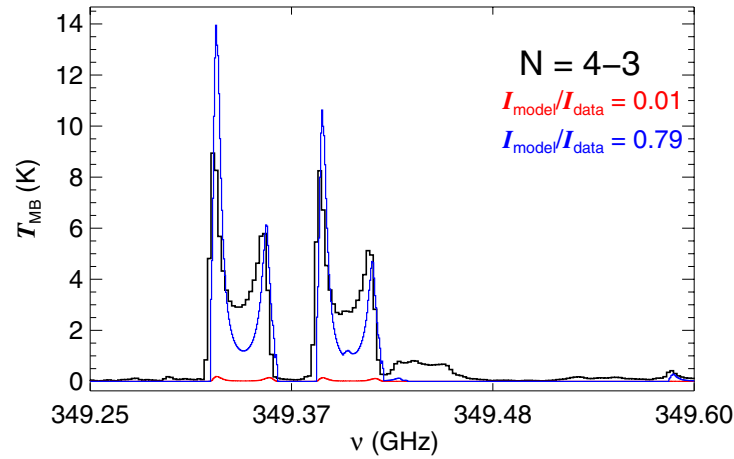

(a) Lines observed with IRAM

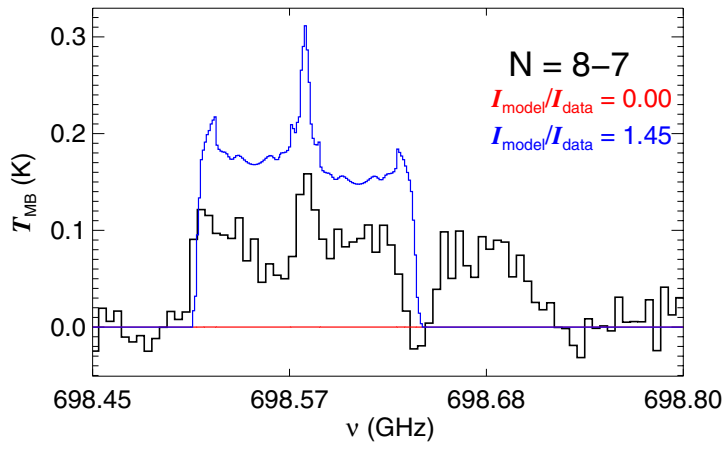

GS

$\mathrm{GS}+v_{2}+v_{3}+v_{1}$

Fig. 13. Comparison of the measured $\mathrm{C}_{2} \mathrm{H}$ spectra (black histograms; see Sect. 2 and Fig. 1 for a description and for the identification of additional spectral features) and GASTRoNOoM model predictions under NLTE conditions for the case where (red) only the ground state is included, and (blue) the ground state, and the three vibrational modes are taken into account. These predictions are based on the "enhanced" abundance profile shown in Fig. 10 and the ${ }^{1} \Sigma$-approximation of $\mathrm{C}_{2} \mathrm{H}$. The transition $N-(N-1)$ and the ratio $I_{\text {model }} / I_{\text {data }}$ are stated in the upper right corner of each panel, according to the colour code of the plots.

(van Dishoeck et al. 2006). Hence, we did not take this $v_{2}$-state population effect into account.

Recently, Tenenbaum et al. (2010) reported on the detection of the $\mathrm{C}_{2} \mathrm{H} N=3-2$ transition in the $v_{2}=$ 1 state, with the Arizona Radio Observatory Submillimeter Telescope (ARO-SMT). Observed peak intensities are of the order of $\sim 5-10 \mathrm{mK}$ in antenna temperature, with reported noise levels around $3 \mathrm{mK}$, and a total velocity-integrated intensity of $\sim 0.2 \mathrm{~K} \mathrm{~km} \mathrm{~s}^{-1}$. The $v_{2}=1, N=3$ level is located at $\sim 560 \mathrm{~K}$ and is not populated under LTE conditions, given the gas-kinetic temperature in the radical shell $(\sim 20 \mathrm{~K})$. Under NLTE conditions, however, the $v_{2}$-levels are easily populated. The prediction of the $v_{2}=1, N=3-2$ emission under the different ${ }^{1} \Sigma$-approximations is shown in Fig. 15. Under the 


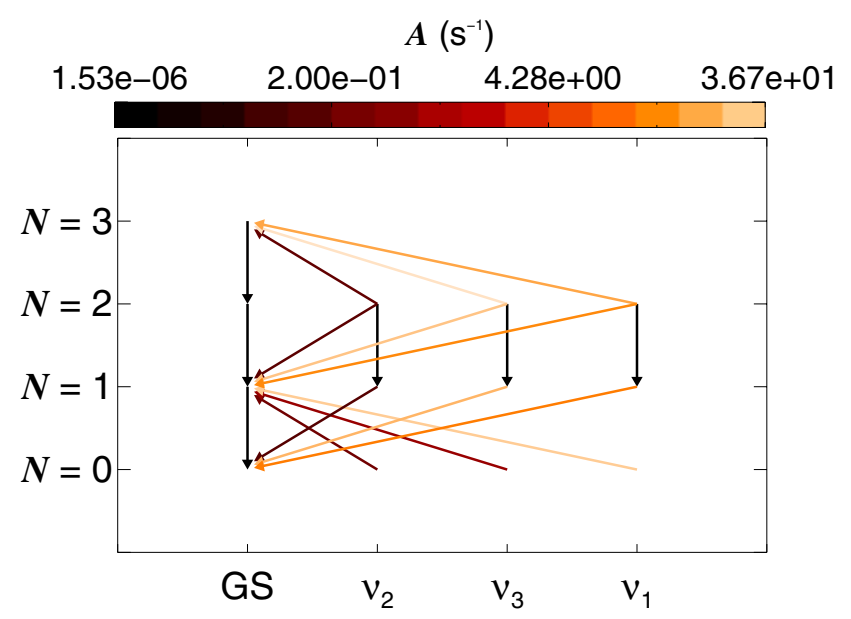

Fig. 14. Visualisation of the transitions responsible for the depopulation of the $N=1$ level in the vibrational ground state (GS), where vibrational states are indicated on the horizontal axis, and rotational levels $N$ on the vertical axis. The colour of the arrows representing the transitions is indicative of the magnitude of the Einstein- $A$ coefficients of these transitions, as given by the colour bar.

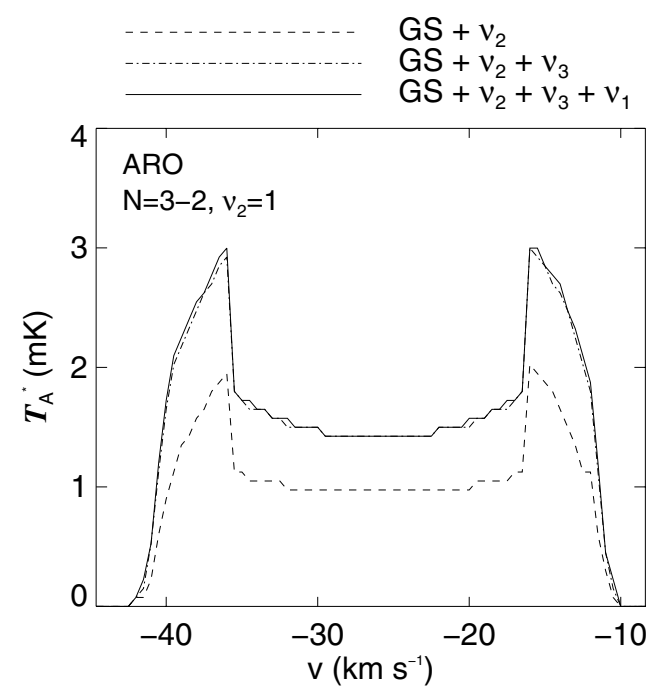

Fig. 15. Predicted line profiles for the $N=3-2$ transition in the $v_{2}=1$ vibrationally excited mode, under assumption of NLTE conditions, and different ${ }^{1} \Sigma$-approximations, as it would be observed with the Arizona Radio Observatory Submillimeter Telescope.

${ }^{1} \Sigma$-approximation, and assuming an HPBW of $29^{\prime \prime}$ at $261 \mathrm{GHz}$ for ARO, we predict a U-shaped profile with peak antenna temperature $\sim 3 \mathrm{mK}$. Taking into account the observed fine structure, we hence predict an integrated intensity that is a factor $\sim 4$ lower than the observed value. Considering the substantial uncertainties (the low signal-to-noise ratio of the ARO observations, and the limited $\mathrm{C}_{2} \mathrm{H}$ approximation in our model), this agreement is satisfactory.

\section{Summary}

We presented new data of $\mathrm{CO}$ and $\mathrm{C}_{2} \mathrm{H}$ obtained with HIFI, PACS, SPIRE and the IRAM $30 \mathrm{~m}$ telescope. High-resolution spectra of $\mathrm{CO}$ transitions up to $J=16-15$, and of $\mathrm{C}_{2} \mathrm{H}$ transitions up to $N=9-8$ were presented and modelled. The HIFI data of both $\mathrm{CO}$ and $\mathrm{C}_{2} \mathrm{H}$ are the first high-frequency-resolution detections of these lines. They were obtained in the framework of a spectral survey of IRC +10216 over the complete frequency range of the HIFI instrument (Cernicharo et al. 2010b and Cernicharo et al., in prep.).

From an SED fit to ISO data, PACS data, and a set of photometric points, covering the wavelength range $0.1-1000 \mu \mathrm{m}$, we obtained a dust-mass-loss rate of $4.0 \times 10^{-8} M_{\odot} \mathrm{yr}^{-1}$ and a luminosity of $11300 L_{\odot}$ at a distance of $150 \mathrm{pc}$. This luminosity value is valid at $\varphi=0.24$, the phase at which the ISO data were obtained. The luminosity is then expected to vary between $6250 \mathrm{~L}_{\odot}$ and $15800 L_{\odot}$ throughout the star's pulsational cycle, which has a period $\sim 649$ days.

In order to model IRC +10216 's wind, we performed the radiative transfer of the dusty component of the CSE consistently with the gas-radiative transfer of CO. The set of 20 highspectral-resolution $\mathrm{CO}$ lines was modelled to constrain the physical parameters of IRC +10216 's CSE. The kinetic temperature throughout the envelope was described by previous results reported by Fonfría et al. (2008) and Decin et al. (2010a), and is now additionally constrained by the combination of all the high-resolution $\mathrm{CO}$ lines. The temperature profile is characterised by $T_{\text {kin }}(r) \propto r^{-0.58}$ for $r \leq 9 R_{\star}, T_{\text {kin }}(r) \propto r^{-0.4}$ for $10 R_{\star} \leq r \leq 65 R_{\star}$, and $T_{\text {kin }}(r) \propto r^{-1.2}$ at larger radii, with an effective temperature $T_{\text {eff }}=2330 \mathrm{~K}$. The derived mass-loss rate is $1.5 \times 10^{-5} M_{\odot} \mathrm{yr}^{-1}$. This is consistent with earlier results (e.g. Cernicharo et al. 2000) and gives a gas-to-dust-mass ratio of 375 , in line with typical values stated for AGB stars (e.g. Ramstedt et al. 2008). Furthermore, we showed a very good agreement between the predictions for $\mathrm{CO}$ lines up to $J=42-41$ and the newly calibrated PACS spectrum of IRC +10216 . It is the first time that such a large coverage of rotational transitions of $\mathrm{CO}$ is modelled with this level of detail.

We extended our envelope model by including episodic mass loss, based on the model of Cordiner \& Millar (2009). This assumption proved very useful in reconciling the modelled $\mathrm{C}_{2} \mathrm{H}$ emission with the PdBI map of the $N=1-0$ transition of $\mathrm{C}_{2} \mathrm{H}$ published by Guélin et al. (1999), and in reproducing the observed line intensities. A decrease of a factor four in the strength of the interstellar UV field also leads to a satisfactory reproduction the PdBI map, but resulted in poorly modelled line intensities. The inclusion of density enhancements in IRC +10216 's CSE is also supported by observational results based on maps of dust-scattered light (Mauron \& Huggins 1999), molecular emisson (Fong et al. 2003), and photometric maps recently obtained with PACS (Decin et al. 2011).

The ground-based observations of $\mathrm{C}_{2} \mathrm{H}$ transitions involve rotational levels up to $N=4$ with energies up to $\sim 17 \mathrm{~cm}^{-1}$, corresponding to temperatures $\sim 25 \mathrm{~K}$. This temperature is close to the gas kinetic temperature at the position of the radical shell $(\sim 20 \mathrm{~K})$. The recent detection of strong $\mathrm{C}_{2} \mathrm{H}$-emission involving levels with energies up to $\sim 150 \mathrm{~K}$, however, calls for an efficient pumping mechanism to these higher levels. Owing to the spectroscopic complexity of $\mathrm{C}_{2} \mathrm{H}$, with the presence of fine structure and hyperfine structure, we approximated the molecule as a ${ }^{1} \Sigma$-molecule, exhibiting pure rotational lines, without splitting. We illustrated that the inclusion of the bending and stretching modes of $\mathrm{C}_{2} \mathrm{H}$ is crucial in the model calculations, since highenergy levels are much more efficiently (radiatively) populated in this case. At this point, we have not yet included overtones of the vibrational states, nor did we treat the resonance between vibrational levels in the electronic ground state and the first electronically excited $A^{2} \Pi$-state. Applying our simplified molecular treatment of $\mathrm{C}_{2} \mathrm{H}$, we can explain the strong intensities of the 
rotational lines in the vibrational ground state, except for the $N=1-0$ transition. We are also able to account for the excitation of the recently observed rotational transition in the $v_{2}=1$ state, showing the strength of our approach.

Acknowledgements. The authors wish to thank B. L. de Vries for calculating and providing dust opacities based on optical constants from the literature. E.D.B. acknowledges financial support from the Fund for Scientific Research Flanders (FWO) under grant number G.0470.07. M.A is supported by a Marie Curie Intra-European Individual Fellowship within the European Community 7th Framework under grant agreement No. 235753. L.D. acknowledges financial support from the FWO. J.C. thanks the Spanish MICINN for funding support under grants AYA2006-14876, AYA2009-07304 and CSD200903004. HSPM is very grateful to the Bundesministerium für Bildung und Forschung (BMBF) for financial support aimed at maintaining the Cologne Database for Molecular Spectroscopy, CDMS. This support has been administered by the Deutsches Zentrum für Luft- und Raumfahrt (DLR). M.G. and P.R. acknowledge support from the Belgian Federal Science Policy Office via de PRODEX Programme of ESA. RSz and MSch ackowledge support from grant N203 581040 of National Science Center. The Herschel spacecraft was designed, built, tested, and launched under a contract to ESA managed by the Herschel/Planck Project team by an industrial consortium under the overall responsibility of the prime contractor Thales Alenia Space (Cannes), and including Astrium (Friedrichshafen) responsible for the payload module and for system testing at spacecraft level, Thales Alenia Space (Turin) responsible for the service module, and Astrium (Toulouse) responsible for the telescope, with in excess of a hundred subcontractors. HIFI has been designed and built by a consortium of institutes and university departments from across Europe, Canada and the United States under the leadership of SRON Netherlands Institute for Space Research, Groningen, The Netherlands and with major contributionÂA as from Germany, France and the US. Consortium members are: Canada: CSA, U. Waterloo; France: CESR, LAB, LERMA, IRAM; Germany: KOSMA, MPIfR, MPS; Ireland, NUI Maynooth; Italy: ASI, IFSI-INAF, Osservatorio Astrofisico di Arcetri-INAF; Netherlands: SRON, TUD; Poland: CAMK, CBK; Spain: Observatorio AstronÃşmico Nacional (IGN), Centro de Astrobiología (CSIC-INTA). Sweden: Chalmers University of Technology - MC2, RSS \& GARD; Onsala Space Observatory; Swedish National Space Board, Stockholm University - Stockholm Observatory; Switzerland: ETH Zurich, FHNW; USA: Caltech, JPL, NHSC. SPIRE has been developed by a consortium of institutes led by Cardiff University (UK) and including Univ. Lethbridge (Canada); NAOC (China); CEA, LAM (France); IFSI, Univ. Padua (Italy); IAC (Spain); Stockholm Observatory (Sweden); Imperial College London, RAL, UCLMSSL, UKATC, Univ. Sussex (UK); and Caltech, JPL, NHSC, Univ. Colorado (USA). This development has been supported by national funding agencies: CSA (Canada); NAOC (China); CEA, CNES, CNRS (France); ASI (Italy); MCINN (Spain); SNSB (Sweden); STFC (UK); and NASA (USA). PACS has been designed and built by a consortium of institutes and university departments from across Europe under the leadership of Principal Investigator Albrecht Poglitsch located at Max-Planck-Institute for Extraterrestrial Physics, Garching, Germany. Consortium members are: Austria: UVIE; Belgium: IMEC, KUL, CSL; France: CEA, OAMP; Germany: MPE, MPIA; Italy: IFSI, OAP/OAT, OAA/CAISMI, LENS, SISSA; Spain: IAC.

\section{References}

Agúndez, M., Cernicharo, J., Guélin, M., et al. 2010, A\&A, 517, L2

Begemann, B., Mutschke, H., Dorschner, J., \& Henning, T. 1994, in Molecules and Grains in Space, ed. I. Nenner, AIP Conf. Ser., 312, 781

Bohren, C. F., \& Huffman, D. R. 1983, Absorption and scattering of light by small particles (Wiley)

Brown, J. M., \& Millar, T. J. 2003, MNRAS, 339, 1041

Cernicharo, J., \& Guélin, M. 1996, A\&A, 309, L27

Cernicharo, J., Kahane, C., Gomez-Gonzalez, J., \& Guélin, M. 1986a, A\&A, 167, L5

Cernicharo, J., Kahane, C., Gomez-Gonzalez, J., \& Guélin, M. 1986b, A\&A, 164, L1

Cernicharo, J., Guélin, M., Menten, K. M., \& Walmsley, C. M. 1987a, A\&A, 181, L1

Cernicharo, J., Guélin, M., \& Walmsley, C. M. 1987b, A\&A, 172, L5

Cernicharo, J., Yamamura, I., González-Alfonso, E., et al. 1999, ApJ, 526, L41

Cernicharo, J., Guélin, M., \& Kahane, C. 2000, A\&AS, 142, 181

Cernicharo, J., Guélin, M., Agúndez, M., et al. 2007, A\&A, 467, L37

Cernicharo, J., Guélin, M., Agúndez, M., McCarthy, M. C., \& Thaddeus, P. 2008, ApJ, 688, L83
Cernicharo, J., Decin, L., Barlow, M. J., et al. 2010a, A\&A, 518, L136 Cernicharo, J., Waters, L. B. F. M., Decin, L., et al. 2010b, A\&A, 521, L8 Cernicharo, J., Agúndez, M., Kahane, C., et al. 2011, A\&A, 529, L3

Cordiner, M. A., \& Millar, T. J. 2009, ApJ, 697, 68

Crosas, M., \& Menten, K. M. 1997, ApJ, 483, 913

De Beck, E., Decin, L., de Koter, A., et al. 2010, A\&A, 523, A18

de Graauw, T., Helmich, F. P., Phillips, T. G., et al. 2010, A\&A, 518, L6

Decin, L., Hony, S., de Koter, A., et al. 2006, A\&A, 456, 549

Decin, L., Agúndez, M., Barlow, M. J., et al. 2010a, Nature, 467, 64

Decin, L., Cernicharo, J., Barlow, M. J., et al. 2010b, A\&A, 518, L143

Decin, L., De Beck, E., Brünken, S., et al. 2010c, A\&A, 516, A69

Decin, L., Royer, P., Cox, N. L. J., et al. 2011, A\&A, 534, A1

Deguchi, S., \& Nguyen-Q-Rieu. 1990, ApJ, 360, L27

Deleon, R. L., \& Muenter, J. S. 1984, J. Chem. Phys., 80, 3992

Dinh-V-Trung \& Lim, J. 2008, ApJ, 678, 303

Draine, B. T. 1978, ApJS, 36, 595

Dumouchel, F., Faure, A., \& Lique, F. 2010, MNRAS, 406, 2488

Fonfría, J. P., Cernicharo, J., Richter, M. J., \& Lacy, J. H. 2008, ApJ, 673, 445

Fong, D., Meixner, M., \& Shah, R. Y. 2003, ApJ, 582, L39

González-Alfonso, E., \& Cernicharo, J. 1999, in ESA Spec. Publ. 427, The

Universe as Seen by ISO, ed. P. Cox, \& M. Kessler, 325

Goorvitch, D., \& Chackerian, Jr., C. 1994, ApJS, 91, 483

Griffin, M. J., Abergel, A., Abreu, A., et al. 2010, A\&A, 518, L3

Groenewegen, M. A. T., Baas, F., de Jong, T., \& Loup, C. 1996, A\&A, 306, 241

Groenewegen, M. A. T., van der Veen, W. E. C. J., \& Matthews, H. E. 1998, A\&A, 338, 491

Groenewegen, M. A. T., Waelkens, C., Barlow, M. J., et al. 2011, A\&A, 526, A162

Guélin, M., Cernicharo, J., Kahane, C., Gomez-Gonzalez, J., \& Walmsley, C. M. 1987, A\&A, 175, L5

Guélin, M., Green, S., \& Thaddeus, P. 1978, ApJ, 224, L27

Guélin, M., Lucas, R., \& Cernicharo, J. 1993, A\&A, 280, L19

Guélin, M., Cernicharo, J., Travers, M. J., et al. 1997, A\&A, 317, L1

Guélin, M., Neininger, N., Lucas, R., \& Cernicharo, J. 1999, in The Physics and Chemistry of the Interstellar Medium, ed. V. Ossenkopf, J. Stutzki, \& G. Winnewisser, 326

Habing, H. J., \& Olofsson, H. 2003, Asymptotic giant branch stars

He, J. H., Dinh-V-Trung, Kwok, S., et al. 2008, ApJS, 177, 275

Huggins, P. J., Olofsson, H., \& Johansson, L. E. B. 1988, ApJ, 332, 1009

Kahane, C., Gomez-Gonzalez, J., Cernicharo, J., \& Guélin, M. 1988, A\&A, 190, 167

Kama, M., Min, M., \& Dominik, C. 2009, A\&A, 506, 1199

Kawaguchi, K., Fujimori, R., Aimi, S., et al. 2007, PASJ, 59, L47

Kramer, C. 1997, Calibration of spectral line data at the IRAM 30m radio telescope, Tech. rep., IRAM

Ladjal, D., Justtanont, K., Groenewegen, M. A. T., et al. 2010, A\&A, 513, A53

Larsson, B., Liseau, R., \& Men'shchikov, A. B. 2002, A\&A, 386, 1055

Leão, I. C., de Laverny, P., Mékarnia, D., de Medeiros, J. R., \& Vandame, B. 2006, A\&A, 455, 187

Le Bertre, T. 1992, A\&AS, 94, 377

Loup, C., Forveille, T., Omont, A., \& Paul, J. F. 1993, A\&AS, 99, 291

Maercker, M., Schöier, F. L., Olofsson, H., Bergman, P., \& Ramstedt, S. 2008, A\&A, 479, 779

Mauron, N., \& Huggins, P. J. 1999, A\&A, 349, 203

Men'shchikov, A. B., Balega, Y., Blöcker, T., Osterbart, R., \& Weigelt, G. 2001, A\&A, 368, 497

Min, M., Hovenier, J. W., \& de Koter, A. 2003, A\&A, 404, 35

Min, M., Dullemond, C. P., Dominik, C., de Koter, A., \& Hovenier, J. W. 2009, A\&A, 497, 155

Monnier, J. D., Danchi, W. C., Hale, D. S., Tuthill, P. G., \& Townes, C. H. 2000 ApJ, 543, 868

Mordaunt, D. H., Ashfold, M. N. R., Dixon, R. N., Löffler, P., Schnieder, L., \& Welge, K. H. 1998, J. Chem. Phys., 108, 519

Müller, H. S. P., Klaus, T., \& Winnewisser, G. 2000, A\&A, 357, L65

Müller, H. S. P., Schlöder, F., Stutzki, J., \& Winnewisser, G. 2005, J. Mol. Struct., 742,215

Olofsson, H., Eriksson, K., Gustafsson, B., \& Carlstrom, U. 1993, ApJS, 87, 267 Padovani, M., Walmsley, C. M., Tafalla, M., Galli, D., \& Müller, H. S. P. 2009, A\&A, 505, 1199

Pilbratt, G. L., Riedinger, J. R., Passvogel, T., et al. 2010, A\&A, 518, L1

Pitman, K. M., Hofmeister, A. M., Corman, A. B., \& Speck, A. K. 2008, A\&A, 483, 661

Poglitsch, A., Waelkens, C., Geis, N., et al. 2010, A\&A, 518, L2

Preibisch, T., Ossenkopf, V., Yorke, H. W., \& Henning, T. 1993, A\&A, 279, 577

Ramstedt, S., Schöier, F. L., Olofsson, H., \& Lundgren, A. A. 2008, A\&A, 487,645

Remijan, A. J., Hollis, J. M., Lovas, F. J., et al. 2007, ApJ, 664, L47 
Ridgway, S., \& Keady, J. J. 1988, ApJ, 326, 843

Roelfsema, P. R., Helmich, F. P., Teyssier, D., et al. 2012, A\&A, 537, A17

Schloerb, F. P., Irvine, W. M., Friberg, P., Hjalmarson, A., \& Hoglund, B. 1983, ApJ, 264, 161

Schöier, F. L., Bast, J., Olofsson, H., \& Lindqvist, M. 2007, A\&A, 473, 871

Skinner, C. J., Justtanont, K., Tielens, A. G. G. M., et al. 1999, MNRAS, 302, 293

Tarroni, R., \& Carter, S. 2004, in (Taylor \& Francis Ltd.), 2167-2179, european Network Theonet II Meeting, Bologna, Italy, Nov., 2003

Tenenbaum, E. D., Dodd, J. L., Milam, S. N., Woolf, N. J., \& Ziurys, L. M. 2010, ApJ, 720, L102
Teyssier, D., Hernandez, R., Bujarrabal, V., Yoshida, H., \& Phillips, T. G. 2006, A\&A, 450, 167

Thaddeus, P., Gottlieb, C. A., Gupta, H., et al. 2008, ApJ, 677, 1132

Tucker, K. D., Kutner, M. L., \& Thaddeus, P. 1974, ApJ, 193, L115

van Dishoeck, E. F., Jonkheid, B., \& van Hemert, M. C. 2006, Faraday Discussions, 133, 231

Wang, Y., Jaffe, D. T., Graf, U. U., \& Evans, II, N. J. 1994, ApJS, 95, 503

Winnewisser, G., Belov, S. P., Klaus, T., \& Schieder, R. 1997, J. Mol. Spec., 184 468

Woon, D. E. 1995, Chem. Phys. Lett., 244, 45 